\title{
Antarctic sponges (Porifera, Demospongiae) of the South Shetland Islands and vicinity. Part II. Poecilosclerida ${ }^{1}$
}

\author{
Maurício Campos ${ }^{2,3}$; Beatriz Mothes ${ }^{2} \&$ Inga L. Veitenheimer Mendes ${ }^{3}$
}

\author{
${ }^{1}$ Contribution number 511 of the Programa de Pós-Graduação em Biologia Animal, Instituto de Biociências, Universidade \\ Federal do Rio Grande do Sul. \\ ${ }^{2}$ Museu de Ciências Naturais, Fundação Zoobotânica do Rio Grande do Sul. Rua Salvador França 1427, 90690-000 Porto \\ Alegre, Rio Grande do Sul, Brasil. E-mail: mrcpoa@hotmail.com; bmothes@fzb.rs.gov.br \\ 3 Programa de Pós-Graduação em Biologia Animal, Universidade Federal do Rio Grande do Sul. Avenida Bento Gonçalves \\ 9500, 91501-970 Porto Alegre, Rio Grande do Sul, Brasil. E-mail: inga.mendes@ufrgs.br
}

\begin{abstract}
In the present study 16 species are registered for the South Shetland Islands and vicinity: Acanthorhabdus fragilis Burton, 1929, Iophon unicornis Topsent, 1907, Clathria (Axosuberites) flabellata (Topsent, 1916), Clathria (Axosuberites) nidificata (Kirkpatrick, 1907), Kirkpatrickia variolosa (Kirkpatrick, 1907), Myxodoryx hanitschi (Kirkpatrick, 1907), lotroata somovi (Koltun, 1964), Tedania (Tedaniopsis) charcoti (Topsent, 1907), Tedania (Tedaniopsis) vanhoffeni (Hentschel, 1914), Tedania (Tedaniopsis) oxeata (Topsent, 1916), Isodictya kerguelenensis (Ridley \& Dendy, 1886), Isodictya Iankesteri (Kirkpatrick, 1907), Isodictya toxophila Burton, 1932, Isodictya bentarti Ríos, Cristobo \& Urgorri, 2004, Latrunculia (Latrunculia) brevis Ridley \& Dendy, 1886 and Latrunculia (Latrunculia) biformis (Kirkpatrick, 1908). Amongst the identified species, five are new occurrences for the studied region, three have their bathymetric limit extended and the others are confirmed for the studied area.

KEY WORDS. Antarctica; new occurrences; PROANTAR; taxonomy.
\end{abstract}

\begin{abstract}
RESUMO. Esponjas Antárticas (Porifera, Demospongiae) das Ilhas Shetland do Sul e áreas próximas. Parte II. Poecilosclerida. No presente estudo 16 espécies são registradas para as Illhas Shetland do Sul e áreas próximas: Acanthorhabdus fragilis Burton, 1929, Iophon unicornis Topsent, 1907, Clathria (Axosuberites) flabellata (Topsent, 1916), Clathria (Axosuberites) nidificata (Kirkpatrick, 1907), Kirkpatrickia variolosa (Kirkpatrick, 1907), Myxodoryx hanitschi (Kirkpatrick, 1907), Iotroata somovi (Koltun, 1964), Tedania (Tedaniopsis) charcoti (Topsent, 1907), Tedania (Tedaniopsis) vanhoffeni (Hentschel, 1914), Tedania (Tedaniopsis) oxeata (Topsent, 1916), Isodictya kerguelenensis (Ridley \& Dendy, 1886), Isodictya lankesteri (Kirkpatrick, 1907), Isodictya toxophila Burton, 1932, Isodictya bentarti Ríos, Cristobo \& Urgorri, 2004, Latrunculia (Latrunculia) brevis Ridley \& Dendy, 1886 e Latrunculia (Latrunculia) biformis (Kirkpatrick, 1908). Dentre as espécies identificadas, cinco são novas ocorrências para a região estudada, três têm seu limite batimétrico ampliado e as demais são confirmadas para a área de estudo.
\end{abstract}

PALAVRAS-CHAVE. Antártica; novas ocorrências; PROANTAR; taxonomia.

The order Poecilosclerida is the most species-rich in the antarctic and subantarctic regions. Most species recently recorded in the region belong to the above-mentioned order (Mothes \& Lerner 1995, Calcinai \& Pansini 2000, Ríos et al. 2004). In the present study, knowledge of the recorded species in the South Shetland Islands and vicinity was amplified through descriptions and illustrations, as well as new records for the geographical and bathymetric distributions of each species.

The descriptions were extended by means of Scanning Electron Microscopy photomicrographs (SEM), which were provided in only a few previous reports.

\section{MATERIAL AND METHODS}

The samples were collected through the Brazilian Antarctic Program (PROANTAR), at the South Shetland Islands and in the Bransfield Strait (Fig. 1). Biological sampling was carried out on board of the R/V "Professor Besnard," from 20 to $412 \mathrm{~m}$ depth, by beam-trawl, otter-trawl, scuba diving and others, unspecified collection methods. The specimens are preserved in $96^{\circ} \mathrm{GL}$ alcohol and deposited in the Porifera Collection of the Museu de Ciências Naturais, Fundação Zoobotânica do Rio Grande do Sul, Porto Alegre, RS, Brazil. 


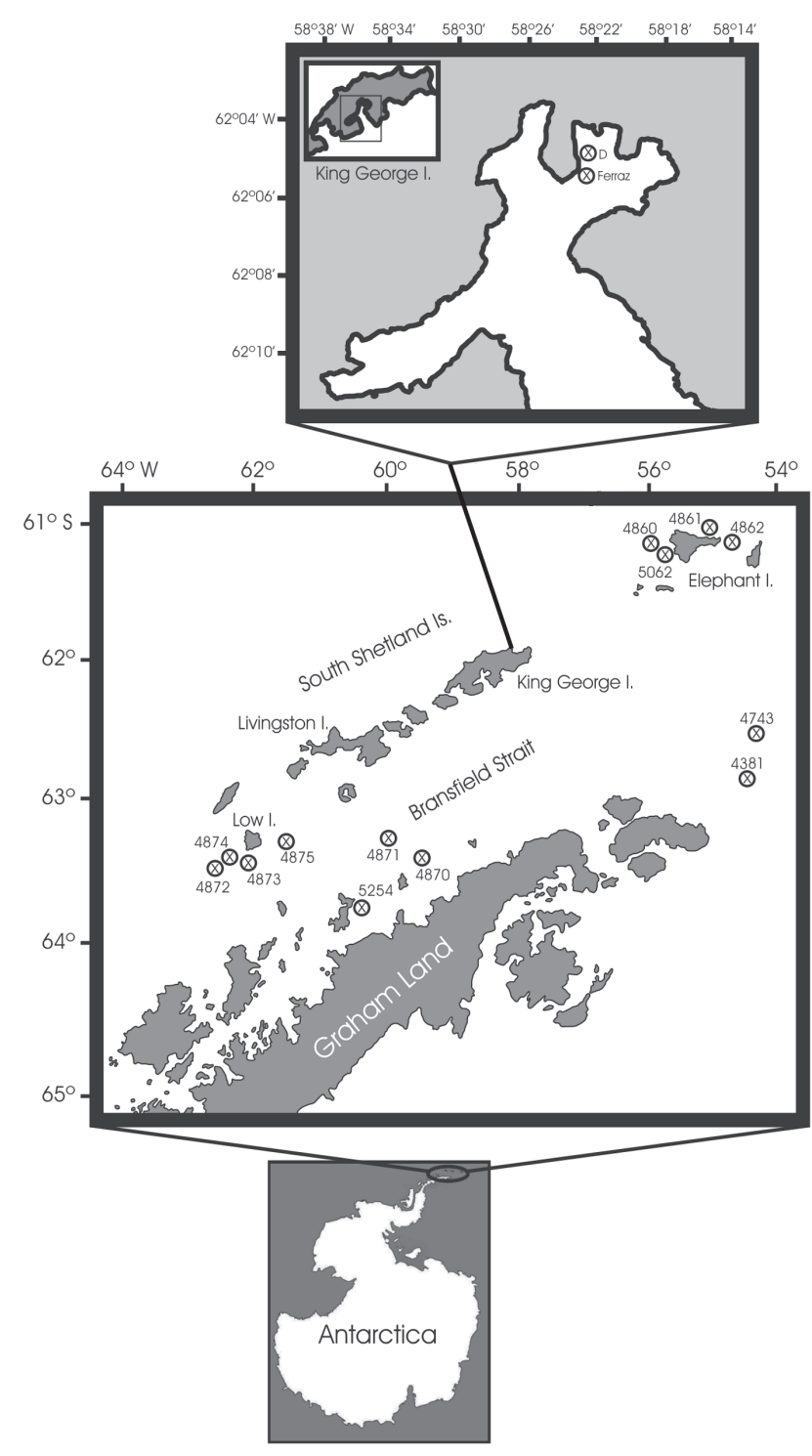

Figure 1. Map showing the collecting area.

Taxonomic study was based on the spicules, by dissociated spicule mounts and thick sections of the skeleton, following Mothes-De-Moraes (1978) and Mothes et al. (2004a), respectively. Preparations for SEM followed SiLva \& Mothes (1996). Spicule measurements comprised minimum, mean, and maximum sizes, width after the bar (/), $\mathrm{N}=50$.

Abbreviations used in the text are: BMNH (The Natural History Museum, London, England), MCNPOR (Porifera Collection, Museu de Ciências Naturais, Fundação Zoobotânica do Rio Grande do Sul, Porto Alegre, RS, Brazil), ZIN (Zoological Institute, Russian Academy of Sciences, Saint Petersburg, Russia) and ZMB (Porifera Collection, Zoologische Museum für Naturkunde an der Universität Humboldt zu Berlin, Berlin, Germany).

\section{RESULTS}

\section{Microcionina Hajdu, Van Soest \& Hooper, 1994 Acarnidae Dendy, 1922 Acanthorhabdus fragilis Burton, 1929}

Figs 2-9, Tab. I

Acanthorhabdus fragilis Burton, 1929: 432, pl. IV, fig. 2, text-fig. 5, 1932: 294, 1938: 11; Koltun, 1964: 58, pl. X, fig. 35-37; Barthel et al., 1990: 122, 1997: 48.

Studied material. MCNPOR 1968, St. 4871, Bransfield Strait: $63^{\circ} 16^{\prime} \mathrm{S}-59^{\circ} 55^{\prime} \mathrm{W}, 264 \mathrm{~m}$, 08.II.1986, PROANTAR IV, "beam-trawl" coll.

Examined material for comparison. Acanthorhabdus fragilis Burton, 1929, collected by the Antarctic Discovery Expedition, locality unknown, slide BMNH 1928.II.15.379.

Description. (Fig. 2) Massive, amorphous specimen; dimensions in cm: 8.6 length, 3.4 width, 2.5 thickness; conulose surface, hispid to the touch; superficial membrane partially broken. Oscules irregularly scattered over the surface, 0.3-0.5 $\mathrm{cm}$ diameter. Preserved material friable in consistency, colour dark brown with clearer regions.

Skeleton. Ectosome formed by a dense tangential arrangement of acanthorhabds (Fig. 3), with loose anisochelas. Choanosome composed of thick tracts of anisoxeas, 10-15 spicules, 350-800 $\mu \mathrm{m}$ thick (Fig. 4), which are interconnected by secondary tracts, 07-10 spicules, 220-400 $\mu \mathrm{m}$ thick. Acanthorhabds and anisochelas scattered along the choanosome.

Spicules. Megascleres: anisoxeas - smooth, slightly curved (Fig. 5); with acerate and conical/mucronate extremities (Fig. 6), in the latter the apical portion can be bifurcate and/or lightly curved; acanthorhabds - spines slightly curved towards the center of the spicule (Fig. 7); both the extremities are composed of 03-05 spines, 3.8-5.2-7.5 $\mu \mathrm{m}$ height (Fig. 8). Microscleres: anisochelas (Fig. 9) - palmate, with a spurred extremity. Measurements (Tab. I).

Remarks. Until now, Acanthorhabdus fragilis has been recorded only from Antarctica. This is the first record of the species for the studied area.

The genus Acanthorhabdus is atypical for the family Acarnidae, because of the morphology of the acanthorhabds and also the reticulation formed by these spicules; however, the presence of spurred chelas, which is shared with the genus Iophon, inserts the taxon in the Acarnidae (HoOper 2002).

The measurements of the spicules from our sample are very similar to those provided by KoLTuN (1964), differing only in respect to the presence of thicker anisoxeas. The comparative

Table I. Acanthorhabdus fragilis, spicules measurements.

\begin{tabular}{cccc}
\hline MCNPOR & Anisoxeas & Acanthorhabds & Anisochelas \\
\hline \multirow{2}{*}{1968} & $380-463.2-500 /$ & $280-322.4-380 /$ & $20-22.8-27.5$ \\
& $25-37.1-50$ & $12.5-18.2-25$ & \\
\hline
\end{tabular}




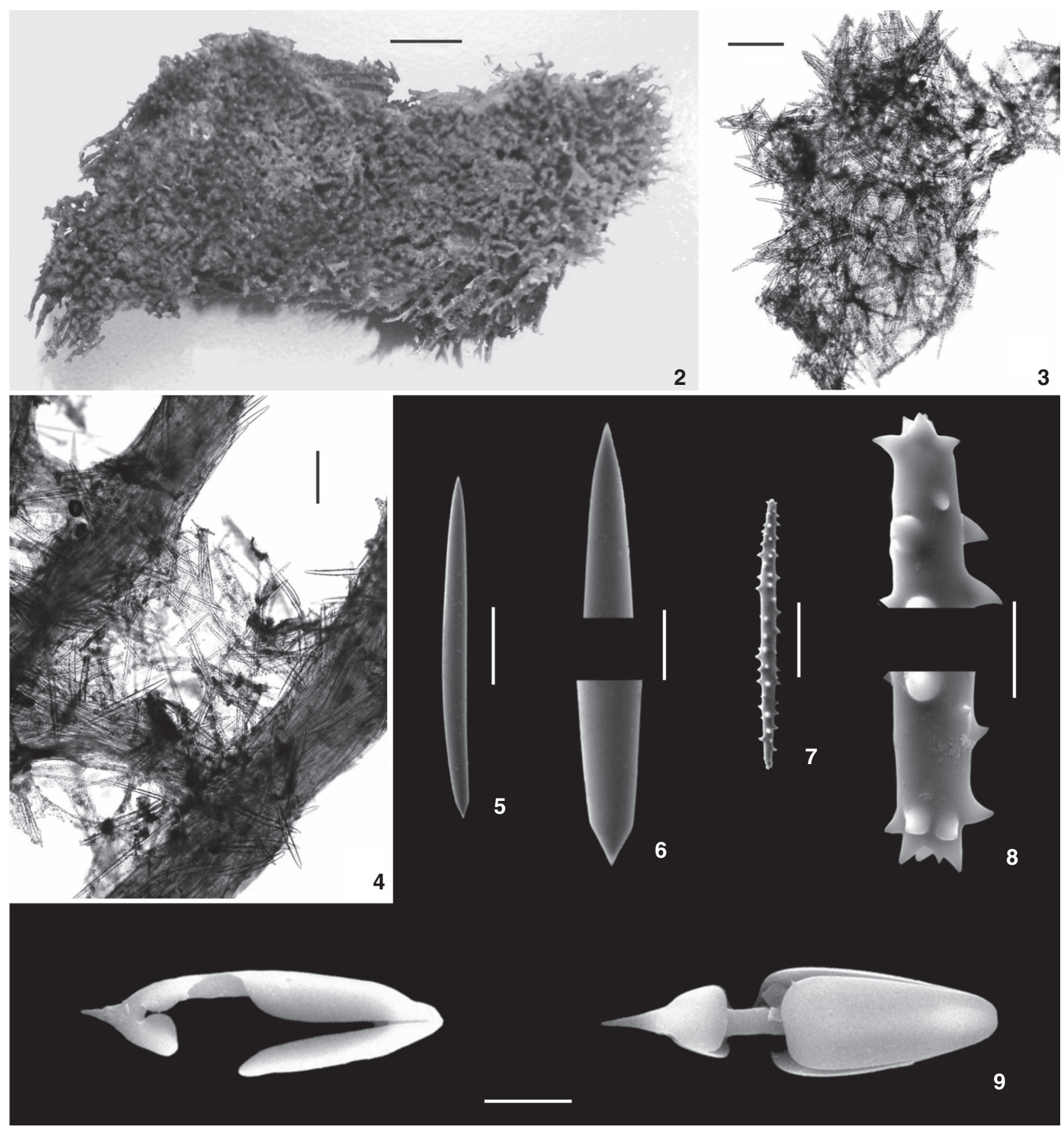

Figures 2-9. Acanthorhabdus fragilis: (2) preserved specimen; (3) ectosomal arrangement of the skeleton; (4) choanosomal skeleton; (5) anisoxea; (6) detail of anisoxea extremities; (7) acanthorhabd; (8) detail of acanthorhabd extremities; (9) anisochelas. Scale bars: (2) 1 cm; (3) $300 \mu \mathrm{m}$; (4) $500 \mu \mathrm{m}$; (5) $100 \mu \mathrm{m}$; (6) $30 \mu \mathrm{m}$; (7) $80 \mu \mathrm{m}$; (8) $10 \mu \mathrm{m}$; (9) $5 \mu \mathrm{m}$.

material has slightly more-swollen anisoxeas, but in general the spiculation concords with the material of the present study. Distribution. Antarctica: Victoria Land (BURTON 1929, 1932,
1938); Wilhelm II Land (Koltun 1964); Weddell Sea (BARThel et al. 1990, 1997); Bransfield Strait (present study). Bathymetry: 72 to 560 m (Koltun 1964).

Revista Brasileira de Zoologia 24 (3): 742-770, setembro 2007 


\section{Iophon unicornis Topsent, 1907 \\ Figs 10-17, Tab. II}

Iophon unicornis Topsent, 1907: 72; Desqueyroux \& Moyano, 1987: 49; Desqueyroux-Faúndez, 1989: 120, pl. III, fig. 17ad, pl. XII, figs 68-70; Pansini et al., 1994: 75; Gutt \& Koltun, 1995: 231; Cattaneo-Vietti et al., 1999: 540.

Iophon spatulatus Kirkpatrick, 1907: 276; Koltun, 1976: 182;

Desqueyroux, 1975: 64, pl. III, figs 36-40; Sarà et al., 1990: 254; Barthel et al., 1997: 48.

Further synonym see Desqueyroux (1975) and DesqueyrouxFAÚNDEZ (1989).

Studied material. MCNPOR 1974, St. 4860, Elephant I.:

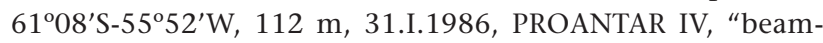
trawl" coll.; MCNPOR 3125, St. 'D', King George I.: $62^{\circ} 05^{\prime} \mathrm{S}$ 58²3’W, $21 \mathrm{~m}$, 08.XII.1989, PROANTAR VIII, "scuba diving" coll.; MCNPOR 1991, 1994, St. 4871, Bransfield Strait: 63016'S5955'W, 264 m, 08.II.1986, PROANTAR IV, “beam-trawl” coll.

Description. (MCNPOR 3125) (Fig. 10) Fragment partially broken; dimensions in cm: 2.3 length, 1.7 width, 0.8 height; slightly conulose surface, on magnifying glass it was observed the formation of small protruding spicule tracts; at apical portion there is an oscular opening $(0.2 \times 0.6 \mathrm{~cm})$, in cylindrical form. Preserved material of fragile consistency, colour dark brown, with some regions in beige.

Skeleton. (Fig. 11) Ectosome without specialization. Choanosome formed by irregular multispicular tracts; between them megascleres are randomly distributed with loose microscleres and a little amount of spongin.

Spicules. Megascleres: styles - smooth, slightly curved, some straight (Fig. 12), basal extremity mucronate, apical extremity acerate (Fig. 13); acanthostrongyles - slightly curved (Fig. 14), one extremity with 07-11 small spines, the other with a well developed spine (Fig. 15). Microscleres: anisochelas (Fig. 16) - palmate, spurred at one extremity; bipocillas (Fig. 17) generally in 'C'Shape, bearing curved spines in both the extremities. Measurements (Tab. II).

Remarks. Topsent (1907), in describing I. unicornis, found no bipocillas. This kind of spicule was observed by KIRKPATRICK (1908) for I. spatulatus, in which their occurrence is rare. Based on the possibility that the two species are very similar, Topsent (1913) advanced the hypothesis that they would be so. This was corroborated by DesqueYroux-Faúndez (1989), who considered that the two are conspecific, and the name I. unicornis has priority.

Certainly the species shows intraspecific variation: in the present study, bipocillas were absent in one sample, and there was slight variation in the extremities of the acanthostrongyles. Accordingly, a generic revision to examine the real limits of this putative variation in the species diagnosis would be useful.

Distribution. Indian Ocean: Heard I. (Koltun 1976). South America: Chile (Desqueyroux \& Moyano 1987); South Orkney I. (Topsent 1913). Antarctica: Graham Land (Topsent 1907, 1908, DesqueYrouX-Faúndez 1989); Victoria Land (KirKPATrick 1907, 1908, SarÀ et al. 1990, Pansini et al. 1994, Cattaneo-VietTi et al. 1999); Wilhelm II Land (Hentschel 1914); Knox Land; Banzare Land; Princess Elisabeth Land; McRobertson Land; Princess Astrid Land (Koltun 1964); Enderby Land; Adelie Land (Vacelet \& Arnaud 1972, Koltun 1976); Weddell Sea (Gutt \& Koltun, 1995; Barthel et al. 1997); South Shetland I.: Deception I. (Desqueyroux 1975); King George I. (Koltun 1964, present study); Elephant I. (present study); Bransfield Strait (present study). Bathymetry: $0 \mathrm{~m}$ (Desqueyroux \& Moyano 1987) to 623 m (Gutt \& Koltun, 1995).

\section{Microcionidae Carter, 1875 Microcioninae Carter, 1875}

\section{Clathria (Axosuberites) flabellata (Topsent, 1916)}

Figs 18-25, Tab. III

Ophlitaspongia flabellata Topsent, 1916: 167.

Axociella flabellata; Koltun, 1964: 70, pl. XII, figs 12-14; Barthel et al., 1990: 123, 1997: 48; Gutt \& Koltun, 1995: 230.

Clathria (Axosuberites) flabellata; Ríos et al. 2004:103, figs 4a-j. Further synonym see Koltun (1964).

Studied material. MCNPOR 3118, St. Ferraz, King George I.: $62^{\circ} 05^{\prime} \mathrm{S}-58^{\circ} 23^{\prime} \mathrm{W}, 20 \mathrm{~m}, 03 . \mathrm{II} .1985$, PROANTAR III, “trap" coll.; MCNPOR 1972, St. 4870, Bransfield Strait: 63²6'S59³2'W, 135 m, 14.II.1986, PROANTAR IV, "beam-trawl" coll.

Description. (MCNPOR 1972) (Fig. 18) Flabellate specimen, with a longer and thin basal portion; dimensions in $\mathrm{cm}$ : 11.2 height, 4.7 width, 0.4 width at the basal portion; surface hispid to the touch, where on magnifying glass it can be observed some protruding spicules; only three oscular openings are present $(0.1 \mathrm{~cm}$ diameter). Preserved material slightly elastic in consistency, colour greyish beige.

Skeleton. (Fig. 19) Ectosome formed by a crust of styles II in confusion, crust $560-800 \mu \mathrm{m}$ thick. In such region it was also observed toxas and some amount of exogenous material.

Table II. Iophon unicornis, spicules measurements.

\begin{tabular}{ccccc}
\hline MCNPOR & Styles & Acanthostrongyles & Anisochelas & Bipocillas \\
\hline 1974 & $480-535-610 / 15-20.6-22.5$ & $270-310.8-350 / 7.5-11-12.5$ & $15-19.6-22.5$ & - \\
1991 & $450-561.4-620 / 12.5-24.5-27.5$ & $260-311-380 / 8.8-12.2-15$ & $16.3-19.6-23.8$ & $8.8-11.2-12.5$ \\
1994 & $480-553-630 / 17.5-24.9-35$ & $230-250-280 / 5.0-7.6-10$ & $16.3-18.6-21.3$ & $10-11.4-13.8$ \\
3125 & $390-472.4-540 / 11.3-18.6-22.5$ & $250-287.8-330 / 5.0-8.8-11.3$ & $18.8-20.6-23.8$ & $10-12.3-13.8$ \\
\hline
\end{tabular}




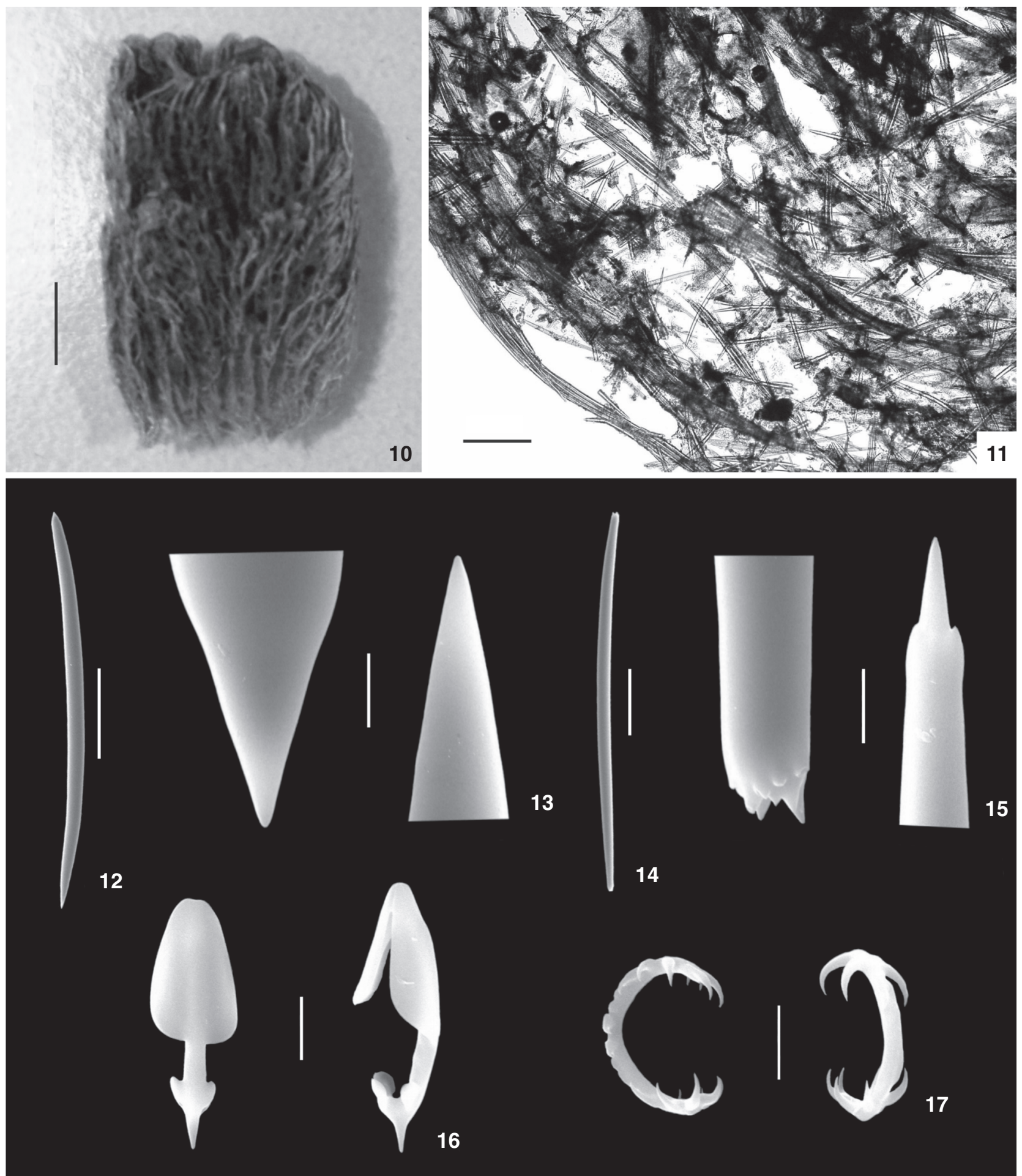

Figures 10-17. lophon unicornis: (10) preserved specimen; (11) skeleton; (12) style; (13) detail of style extremities; (14) acanthostrongyle; (15) detail of acanthostrongyle extremities; (16) anisochelas; (17) bipocillas. Scale bars: (10) $0.5 \mathrm{~cm}$; (11) $500 \mu \mathrm{m}$; (12) $100 \mu \mathrm{m}$; (13) 5 $\mu \mathrm{m}$; (14) $50 \mu \mathrm{m}$; (15) $10 \mu \mathrm{m}$; (16), (17) $5 \mu \mathrm{m}$.

Revista Brasileira de Zoologia 24 (3): 742-770, setembro 2007 

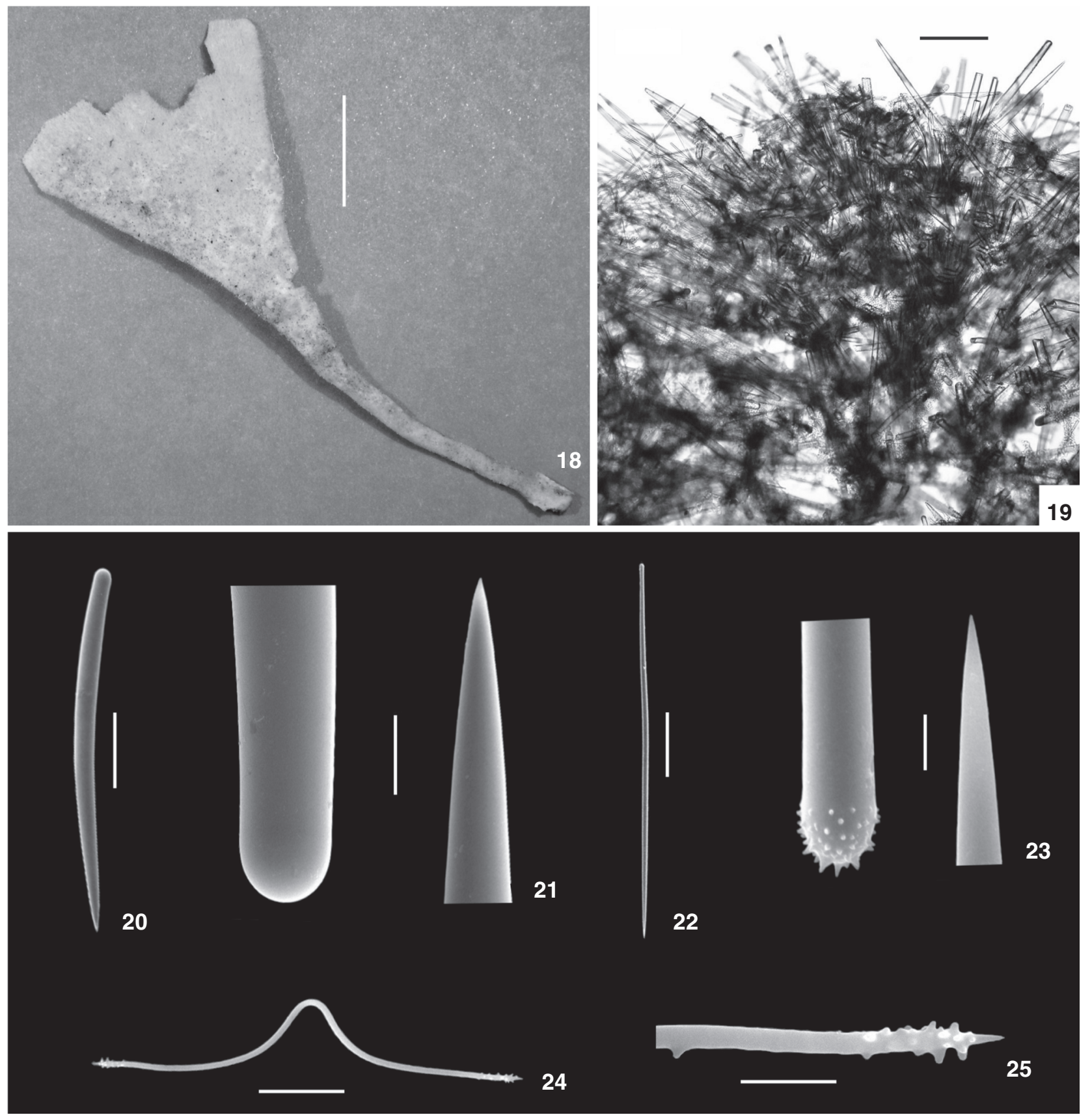

Figures 18-25. Clathria (Axosuberites) flabellata:. (18) preserved specimen; (19) skeleton; (20) style I; (21) detail of style I extremities; (22) style II; (23) detail of style II extremities; (24) toxa; (25) detail of toxa extremity. Scale bars: (18) $2 \mathrm{~cm}$; (19) $500 \mu \mathrm{m}$; (20) $100 \mu \mathrm{m}$; (21) $20 \mu \mathrm{m}$; (22) $100 \mu \mathrm{m}$; (23), $5 \mu \mathrm{m}$; (24), $20 \mu \mathrm{m}$; (25) $5 \mu \mathrm{m}$.

Choanosome made of styles I, condensed in an axial arrangement.

Spicules. Megascleres: styles I - smooth, curved, some sinuous (Fig. 20), apical extremity acerate (Fig. 21); styles II straight (Fig. 22), basal portion bearing small spines (Fig. 23).
Microscleres: toxas - opening angle varies slightly (Fig. 24); spined extremities (Fig. 25). Measurements (Tab. III).

Remarks. Based on the literature, the toxas show some differences in regard to size classes. Topsent (1917) and Koltun (1964) recorded only one size class; on the other hand, Ríos et al.

Revista Brasileira de Zoologia 24 (3): 742-770, setembro 2007 
Table III. Clathria (Axosuberites) flabellata, spicules measurements.

\begin{tabular}{cccc}
\hline MCNPOR & Styles I & Styles II & Toxas \\
\hline \multirow{2}{*}{1972} & $420-688.4-1050 /$ & $380-579-750 /$ & $80-153.4-220 /$ \\
& $25-36.2-53.8$ & $2.5-7.4-12.5$ & $<2.5$ \\
\multirow{2}{*}{3118} & $470-740.2-1250 /$ & $340-579.4-850 /$ & $75-133.6-247.5 /$ \\
& $17.5-32.1-57.5$ & $2.5-7.9-13.8$ & $<2.5$ \\
\hline
\end{tabular}

(2004) reported toxas in three categories. In the present study, it was impossible to separate them into classes, because even though the size varied widely, there were no clear limits for categories. The presence of toxas in a wider size variation reported by Ríos et al. (2004) perhaps made possible this differentiation.

The synonymy with C. nidificata (Kirkpatrick, 1907), proposed by Koltun (1976) and Desqueyroux (1975), also including C. rameus Koltun, 1964, may not be confirmed by more detailed analysis of these species. Their spiculation clearly differs, in regard to the dimensions of the styles and mainly of the toxas. The external morphology of the three species also seems to characterize different groups: C. flabellata is flabellate, $C$. nidificata is arborescent with coalescent branches and C. rameus is composed of a single branch.

Distribution. South America: South Georgia (BurTon 1932, 1934). Antarctica: Graham Land (Topsent 1916, 1917); Ross Sea (Burton 1929); Clarie Land; McRobertson Land; Victoria Land (Koltun 1964); Weddell Sea (Barthel et al. 1990, 1997, GutT \& Koltun 1995); Bransfield Strait (present study); South Shetland I.: Livingston I. (Ríos et al. 2004); King George I. (present study). Bathymetry: $20 \mathrm{~m}$ (Ríos et al. 2004, present study) to $700 \mathrm{~m}$ (Koltun 1964).

\section{Clathria (Axosuberites) nidificata (Kirkpatrick, 1907) Figs 26-35, Tab. IV}

Ophlitaspongia nidificata Kirkpatrick, 1907: 274.

Axociella nidificata; Burton, 1940: 116; Koltun, 1964: 70, pl. XII, figs 7-11, 1976: 190; Desqueyroux, 1975: 67; Sarà et al., 1990: 254; Barthel et al., 1990: 123, 1997: 48; Pansini \& Sarà, 1999: 205; Cattaneo-Vietti et al., 1999: 540.

Clathria (Axociella) nidificata; Mothes \& Lerner, 1995: 159, figs 22-27, 55.

Further synonym see BurTon (1940).

Studied material. MCNPOR 1969, St. 4874, Bransfield Strait: $63^{\circ} 25^{\prime} \mathrm{S}-62^{\circ} 19^{\prime} \mathrm{W}, 135 \mathrm{~m}, 14 . \mathrm{II} .1986$, PROANTAR IV, "beam-trawl" coll.; MCNPOR 1993, St. 4872, Bransfield Strait: $63^{\circ} 28^{\prime} \mathrm{S}-62^{\circ} 31^{\prime} \mathrm{W}, 168 \mathrm{~m}, 13 . \mathrm{II} .1986$, PROANTAR IV, “beamtrawl" coll.

Examined material for comparison. Clathria (Axosuberites) nidificata (Kirkpatrick, 1907), collected by the Brazilian Antarctic Program, locality: Joinville I., MCNPOR 1144 (identified by B. Mothes \& C. B. Lerner).

Description. (MCN 1969) (Fig. 26) Palmate specimen, constituted by three main branches, two of them bifurcated and anastomosed at the terminal portion; dimensions in $\mathrm{cm}$ : 8.4 height, 6.2 width, branches ranging from 1.2-1.5 diameter; surface hispid to the touch, with thin erect conules regularly distributed; oscules not visualized. Preserved material slightly hard, with incompressible consistency, colour beige with greyish regions.

Skeleton. (Fig. 27) Ectosome with no specialization, with a discrete agglomeration of toxas I and II. Choanosome with an axial condensation, originating from a 'center' Where megascleres are arranged in confusion; multispicular tracts runs towards the surface, $140-230 \mu \mathrm{m}$ thick. Between the tracts there are free spicules and a little amount of spongin.

Spicules. Megascleres: styles I - smooth, straight to curved (Fig. 28), apical extremity acerate (Fig. 29); styles II - straight, tornote-like (Fig. 30), with rounded spined extremities (Fig. 31). Microscleres: toxas I - microspined extremities (Figs 32, 33), some of them slightly twisted; toxas II - similar to general morphology of toxas I (Figs 34, 35). Measurements (Tab. IV).

Remarks. In our samples, two size classes of toxas could be distinguished, mainly by the width and by the presence of well-developed spines at the extremities of toxas I. The measurements of styles I are larger than those given in previous records of the species. However, considering only one valid taxon rather than three (see remarks on C. flabellata), some records cannot supply the characteristics that truly belong to the species proposed here.

Distribution. South America: Argentina (BURTON 1940); Magellan Strait (PAnsini \& SARÀ 1999); South Georgia (BurToN 1932). Antarctica: Victoria Land (KirKPATRICK 1907, 1908, Burton 1929, SarÀ et al. 1990, Cattaneo-Vietti et al. 1999); Clarie Land, McRobertson Land (Koltun 1964); Enderby Land (KoltuN 1976); Weddell Sea (BARTHEL et al. 1990, 1997); South Shetland I.: Greenwich I. (Desqueyroux 1975); Elephant I. (Mothes \& Lerner 1995), King George I. (Koltun 1964); Bransfield Strait (present study); Joinville I. (Mothes \& Lerner 1995). Bathymetry: 84 m (Burton 1940) to 540 m (Burton 1929).

\section{Myxillina Hajdu, Van Soest \& Hooper, 1994 Hymedesmiidae Topsent, 1928 Kirkpatrickia variolosa (Kirkpatrick, 1907) Figs 36-41, Tab. V}

Tedania variolosa Kirkpatrick, 1907: 279.

Kirkpatrickia variolosa; Koltun, 1976: 182; Barthel et al., 1990: 122, 1997: 48.

Further synonym see Koltun (1976).

Studied material. MCNPOR 1947, St. 4870, Bransfield Strait: 6326'S-59³2'W, 135 m, 08.II.1986, PROANTAR IV, “beamtrawl" coll.; MCNPOR 3140, St. 5254, Bransfield Strait: 63²4'S$60^{\circ} 25^{\prime} \mathrm{W}, 108$ m, 25.I.1988, PROANTAR VI, "otter-trawl" coll.

Description. (MCNPOR 1947) (Fig. 36) Globose specimen; dimensions in cm: 7.5 height, 10.2 length, 7.7 width; conulose surface, due to some skeletal features presented by spicule tracts; 


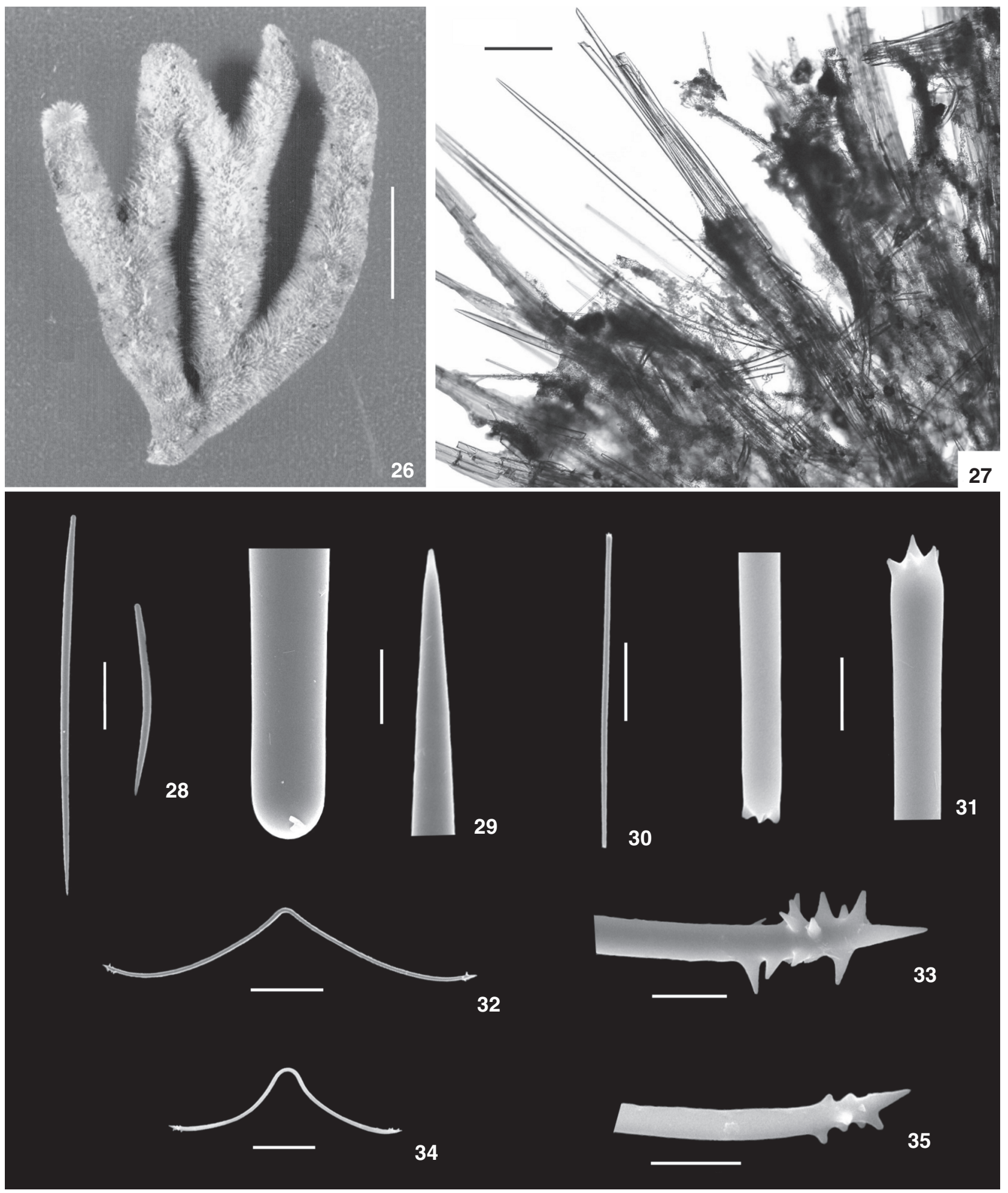

Figures 26-35. Clathria (Axosuberites) nidificata: (26) preserved specimen; (27) skeleton; (28) style I; (29) detail of style I extremities; (30) style II; (31) detail of style II extremities; (32) toxa I; (33) detail of toxa I extremity; (34) toxa II; (35) detail of toxa II extremity. Scale bars: (26) $2 \mathrm{~cm}$; (27) $500 \mu \mathrm{m}$; (28) $300 \mu \mathrm{m}$; (29) $20 \mu \mathrm{m}$; (30) $75 \mu \mathrm{m}$; (31) $7 \mu \mathrm{m}$; (32) $120 \mu \mathrm{m}$; (33) $10 \mu \mathrm{m}$; (34) $25 \mu \mathrm{m}$; (35) $5 \mu \mathrm{m}$. 


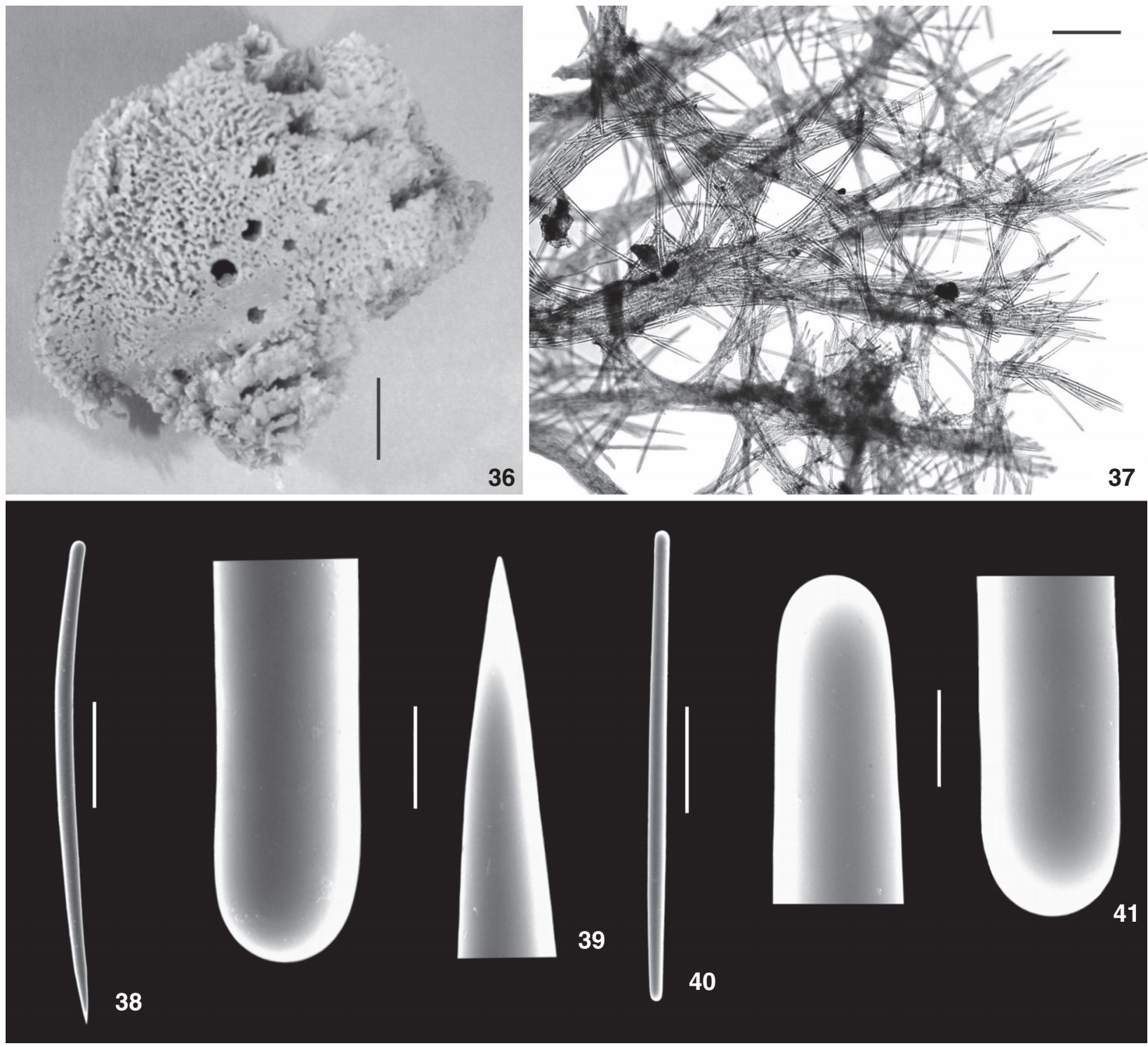

Figures 36-41. Kirkpatrickia variolosa: (36) preserved specimen; (37) skeleton; (38) style; (39) detail of style extremities; (40) tornote; (41) detail of tornote extremities. Scale bars: (36) $2 \mathrm{~cm}$; (37) $500 \mu \mathrm{m}$; (38) $100 \mu \mathrm{m}$; (39) $10 \mu \mathrm{m}$; (40) $50 \mu \mathrm{m}$; (41) $5 \mu \mathrm{m}$.

Table IV. Clathria (Axosuberites) nidificata, spicules measurements.

\begin{tabular}{ccccc}
\hline MCNPOR & Styles I & Styles II & Toxas I & Toxas II \\
\hline 1969 & $520-1173.6-2240 / 22.5-37.5-47.5$ & $280-392.8-570 / 3.8-6.0-8.8$ & $310-564.2-810 / 3.8-5.7-8.8$ & $95-164-275 /<2.5$ \\
1993 & $580-1276.4-2740 / 27.5-40.6-53.8$ & $280-411-600 / 3.8-6.4-8.8$ & $285-455.2-712.5 / 2.5-5.3-9.2$ & $78.2-144.2-230 /<2.5$ \\
\hline
\end{tabular}

Table V. Kirkpatrickia variolosa, spicules measurements.

\begin{tabular}{ccc}
\hline MCNPOR & Styles & Tornotes \\
\hline \multirow{2}{*}{1947} & $380-431.9-475 /$ & $209-266.6-304 /$ \\
& $12.7-14.8-18.4$ & $6.9-7.7-9.2$ \\
\multirow{2}{*}{3140} & $323-397.7-446.5 /$ & $199.5-249.5-285 /$ \\
& $11.5-15.7-18.4$ & $5.8-7.2-8.1$ \\
\hline
\end{tabular}

oscules amongst the conules ( $<0.1-0.5 \mathrm{~cm}$ diameter). Preserved material compressible, slightly fragile, colour beige.

Skeleton. (Fig. 37) Ectosome with tornotes arranged in brushes, 12-18 spicules. Choanosome formed by multispicular ascending tracts of styles, $80-220 \mu \mathrm{m}$ thick; tracts are irregularly connected, both by spicules (in such case perpendicular

Revista Brasileira de Zoologia 24 (3): 742-770, setembro 2007 
to the surface) and by the bifurcation of the tracts.

Spicules. Megascleres: styles - slightly curved, some straight or slightly sinuous (Fig. 38); basal extremity sometimes with small lobular salience, apical extremity varying from acerate to conical (Fig. 39); tornotes - smooth, straight, some slightly curved (Fig. 40), one of the halves can be a little more swelled; blunt extremities (Fig. 41). Measurements (Tab. V).

Remarks. The spiculation of the specimens analysed shows almost no morphological variation in regard to previously published data (KirKPatrick 1908, Koltun 1964).

The chemical composition of $K$. variolosa has stimulated important studies in this field. McClintock \& GAUTHIER (1992) showed that an extract from K. variolosa inhibits the development of certain bacteria. Perry et al. (1994) isolated a compound (variolin B) that shows antitumor and antiviral properties. TRIMURTulu et al. (1994) isolated other compounds (variolin A and $\mathrm{N}\left(3^{\prime}\right)$-methyl tetrahydrovariolin $\mathrm{B}$ ), the latter showing antifungal activity and moderate cytotoxicity. JAYATILAKE et al. (1995) examined this conspicuous red sponge, isolating and identifying a new stilbene derivative, which showed varied biological and pharmacological activity.

Distribution. South America: South Georgia (BurTON 1932). Antarctica: Victoria Land (KirKPATrick 1907, 1908); McRobertson Land (Koltun 1964); Adelie Land; Wilhelm II Land; Enderby Land (Koltun 1976); Weddell Sea (BARThel et al. 1990, 1997); Bransfield Strait (BURTON 1932, present study). Bathymetry: 18 m (KirKPATRICK 1907) to 640 m (Koltun 1976).

\section{Myxodoryx hanitschi (Kirkpatrick, 1907) Figs 42-49, Tab. VI}

Lissomyxilla hanitschi KIRKPATRICK 1907: 336.

Myxodoryx hanitschi; Koltun, 1964: 76, 1976: 182; DesqueyrouxFaúndez, 1989: 115, pl. III, figs 13a-d, pl. X, fig. 57; Barthel et al., 1990: 123; Pansini et al., 1994: 76, pl. VII, figs 2a-c; Cattaneo-Vietti et al., 1999: 540.

Further synonym see Koltun (1964).

Studied material. MCNPOR 1956, St. 4875, Bransfield Strait: 63ำ $17^{\prime} \mathrm{S}-62^{\circ} 30^{\prime} \mathrm{W}, 157 \mathrm{~m}, 14 . \mathrm{I} .1983$, PROANTAR IV, "beam-trawl" coll.

Description. (Fig. 42) Flabellate specimen; dimensions in cm: 12.6 length, 9.5 width, 2.2 height; smooth surface; rounded oscular openings, 0.1-0.4 cm diameter, randomly distributed along the surface, some of them can be often obstructed by a thin dermal membrane. Preserved material slightly compressible, colour beige.

Skeleton. (Fig. 43) Ectosome with no specialization. Choanosome a dense arrangement of longitudinal multispicular tracts, irregularly connected by grouped spicules, together with abundant spongin, forming rounded openings. Tracts running toward the surface, where it forms the superficial hispidation together with free spicules. Equination of the tracts by the acanthostyles was not observed.

Spicules. Megascleres: styles - slightly curved, some
Table VI. Myxodoryx hanitschi, spicules measurements.

\begin{tabular}{cccc}
\hline MCNPOR & Styles & Acanthostyles & Tornotes \\
\hline \multirow{2}{*}{1956} & $350-398.4-460 /$ & $128.8-182.6-225.4 /$ & $260-292-330 /$ \\
& $15-17.9-21.3$ & $4.6-6.9-9.2$ & $7.5-8.3-10$ \\
\hline
\end{tabular}

straight or sinuous (Fig. 44), most of them with no spination or scarcely spined, varying along the shaft, more concentrated at the apical portion; apical extremity acerate (Fig. 45); acanthostyles - straight or slightly curved (Fig. 46), microspination more concentrated towards to both extremities; some with few spines (Fig. 47); tornotes - straight, some sinuous, central portion slightly swollen (Fig. 48); slightly unequal extremities, being one of them acerate and the other mucronate (Fig. 49). Measurements (Tab. VI).

Remarks. Until now, the species is endemic for Antarctica, with its distribution extended in that continent with the present record from the study area.

Its spicules show some differences in regard to previous records for the species; the three kinds of spicules have, in this study, lower limits with smaller values in comparison to published data (Kirkpatrick 1908, Hentschel 1914, Topsent 1917, Burton 1938, Koltun 1976, Desqueyroux-Faúndez 1989, Pansini et al. 1994). In regard to the morphology of the spicules, some styles bear spines, although most have a totally smooth shaft. Some acanthostyles have the spines regularly distributed along the shaft, but most bear spines mainly at their extremities; tornotes have slightly unequal extremities, but the mucronate pattern was also observed. DesQUeYrouX-FAúNDEZ (1989) observed a discrete spination near the basal extremity.

Our SEM microphotographs of the spicules allowed better observation of details, aiding in emending the species diagnosis.

Distribution. Antarctica: Victoria Land (KirKPATRICK 1907, 1908, PAnsini et al. 1994); Graham Land (Topsent 1917, Desqueyroux-Faúndez 1989); Wilhelm II Land (Hentschel 1914); Queen Mary Land (BurTon 1938); Princess Elisabeth Land (Koltun 1976); Bransfield Strait (present study). Bathymetry: 20 m (Desqueyroux-Faúndez 1989) to 622 m (Burton 1938).

\section{lotrochotidae Dendy, 1922 Iotroata somovi (Koltun, 1964)}

Figs 50-57, Tab. VII

Iotrochota somovi Koltun, 1964: 52, text-fig. 12; Barthel et al, 1990: 122; Gutt \& Koltun, 1995: 231.

Iotaota somovi; Koltun, 1976: 182.

Studied material. MCNPOR 2018, St. 4861, Elephant I.: $61^{\circ} 02^{\prime} \mathrm{S}-54^{\circ} 55^{\prime} \mathrm{W}, 362 \mathrm{~m}, 01 . \mathrm{II} .1986$, PROANTAR IV, “beamtrawl" coll.

Examined material for comparison. Iotroata somovi (Koltun, 1964), collected by the Soviet Antarctic Expedition, locality: Banzare Land, Antarctica, slide ZIN 6350 (holotype). 


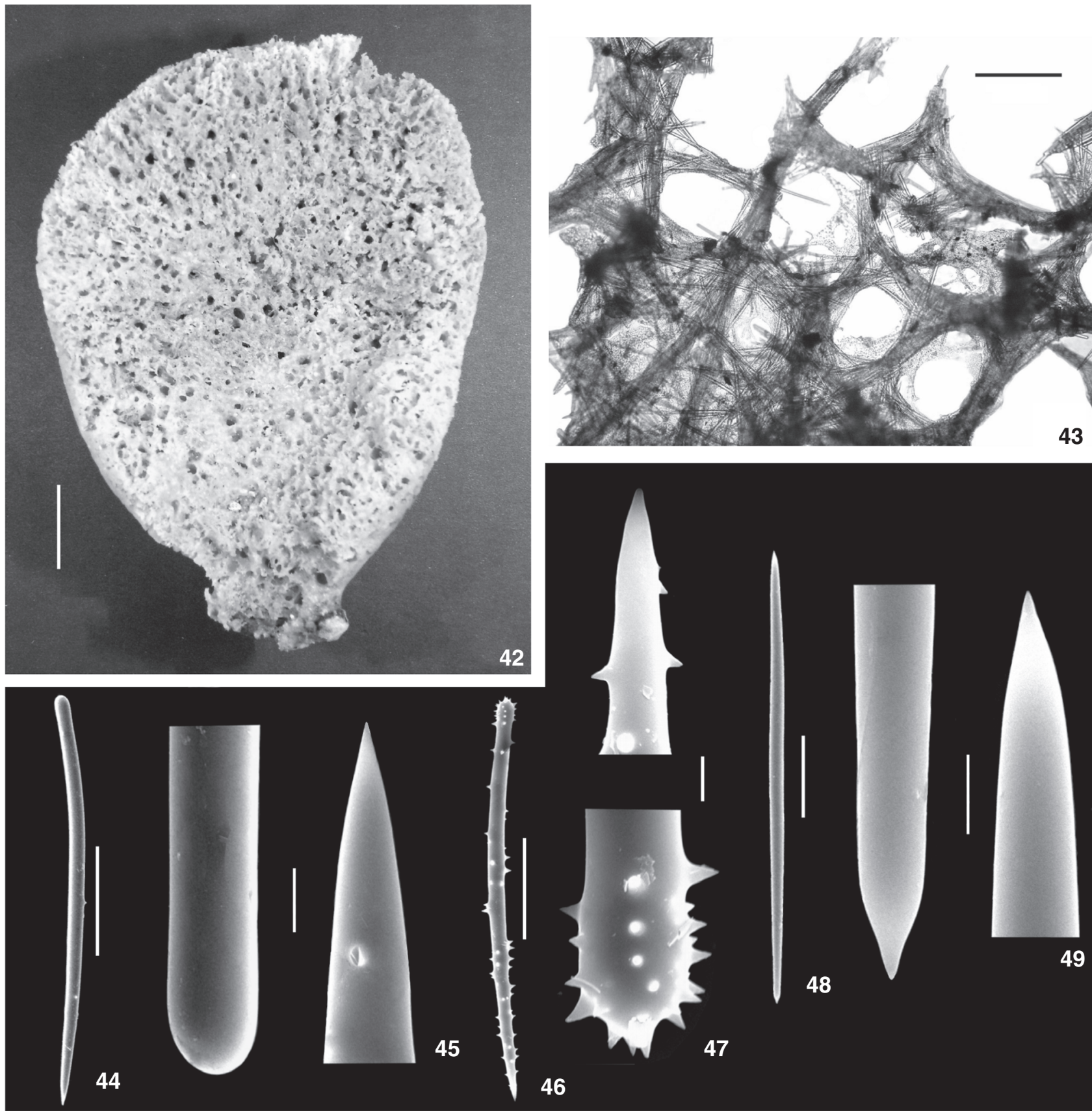

Figures 42-49. Myxodoryx hanitschi: (42) preserved specimen; (43) skeleton; (44) style; (45) detail of style extremities; (46) acanthostyle; (47) detail of acanthostyle extremities; (48) tornote; (49) detail of tornote extremities. Scale bars: (42) $2 \mathrm{~cm}$; (43) $500 \mu \mathrm{m}$; (44) $100 \mu \mathrm{m}$; (45) $10 \mu \mathrm{m}$; (46) $50 \mu \mathrm{m}$; (47) $5 \mu \mathrm{m}$; (48) $50 \mu \mathrm{m}$; (49) $5 \mu \mathrm{m}$.

Table VII. lotroata somovi, spicules measurements.

\begin{tabular}{ccccc}
\hline & Styles & Tylotes & Isochelas & Birotulas \\
\hline MCNPOR 2018 & $610-662.6-730 / 30-36.7-45$ & $240-307-340 / 8.8-10.2-12.5$ & $65-79.1-95$ & $22.5-29.9-40$ \\
ZIN 6350 & $541.5-611.8-684 / 19.6-25.3-31.1$ & $275.5-335-380 / 8.1-10.2-12.7$ & $41.4-52.3-59.8$ & $19.6-23.9-28.8$ \\
\hline
\end{tabular}

Revista Brasileira de Zoologia 24 (3): 742-770, setembro 2007 

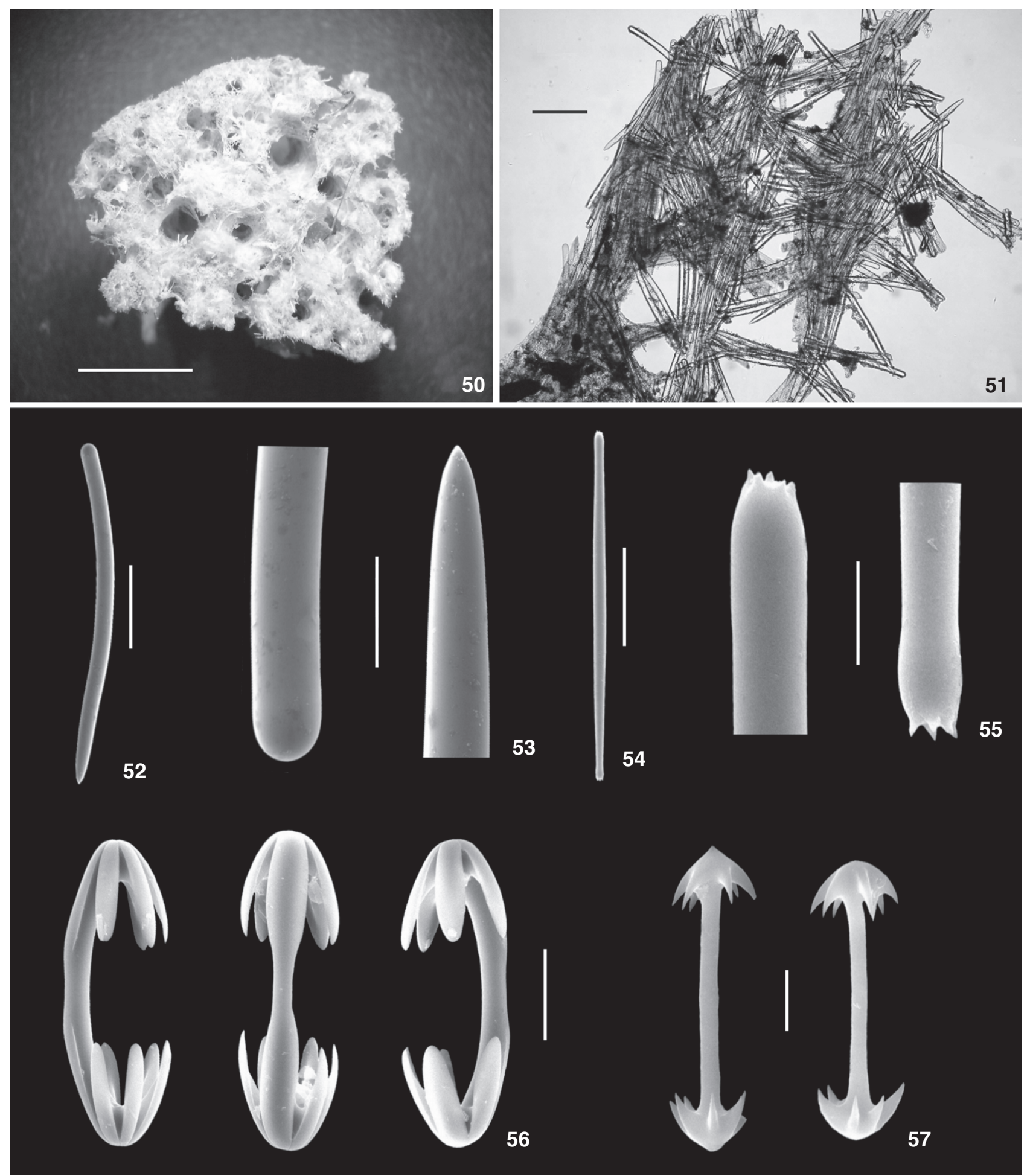

Figures 50-57. lotroata somovi: (50) preserved specimen; (51) skeleton; (52) style; (53) detail of style extremities; (54) tylotes; (55) detail of tylote extremities; (56) isochelas; (57) birotulas. Scale bars: (50) $0.5 \mathrm{~cm}$; (51) $500 \mu \mathrm{m}$; (52) $150 \mu \mathrm{m}$; (53) $50 \mu \mathrm{m}$; (54) $75 \mu \mathrm{m}$; (55) 10 $\mu \mathrm{m}$; (56) $20 \mu \mathrm{m}$; (57) $5 \mu \mathrm{m}$. 
Description. (Fig. 50) Fragment, dimensions in cm: 1.7 length, 1.5 width, 1.0 height; slightly hispid surface, on magnifying glass it can be observed some protruding spicules and conules, this latter bonded by spongin; pores and/or oscules distributed by all over the surface, $0.1-0.3 \mathrm{~cm}$ diameter. Preserved material firm in consistency, colour beige.

Skeleton. (Fig. 51) Ectosomal features were not observed. Choanosome with coarse longitudinal multispicular tracts, 7 11 spicules, 250-370 $\mu \mathrm{m}$ thickness. The tracts are interconnected by irregularly disposed spicules, forming openings at diverse angles. Spongin present mainly between the tracts; microscleres dispersed by the choanosome.

Spicules. Megascleres: styles - smooth, slightly curved, some straight or sinuous (Fig. 52); apical extremity conical, some rounded (Fig. 53); tylotes - smooth, straight (Fig. 54), slightly swollen extremities, presenting 05-08 small spines (Fig. 55). Microscleres: isochelas (Fig. 56) - anchorate, well developed free alae; birotulas (Fig. 57) - extremities bearing spines (05-07), curved and acerate ends. Measurements (Tab. VII).

Remarks. Up to the present, this species is considered endemic to the Antarctic continent. I. somovi does not have a wide geographic distribution; this record is the first for the study area. However, its bathymetric range is wide (for details, see distribution, below).

Spicule measurements were reported previously only by Koltun (1964). Comparative material is morphologically identical to the studied material (remeasurement: Table VII). The sample from the present study has larger dimensions for the styles, birotulas and isochelas, consequently amplifying the description of the spicules; SEM illustrations made it possible for the first time to observe details of the spicules, especially the microscleres.

Distribution. Antarctica: Banzare Land (Koltun 1964); Enderby Land; Sabrina Land (Koltun 1976); Weddell Sea (BARTHEL et al. 1990; GutT \& Koltun 1995); South Shetland I.: Elephant I. (present study). Bathymetry: 208 m (GutT \& Koltun 1995) to 2267 m (Koltun 1976).

\section{Tedaniidae Ridley \& Dendy, 1886 Tedania (Tedaniopsis) charcoti (Topsent, 1907) Figs 58-68, Tab. VIII}

Tedania charcoti Topsent, 1907: 69; Burton, 1940: 106; Lévi, 1964: 149, text-fig. 1, pl. I, fig. 1; Koltun, 1964: 60, pl. X, figs 31-34, 1976: 184; Desqueyroux, 1976: 103; Sarà, 1978: 49, 1991: 233; Cuartas, 1986: 46, 1992: 79: Desqueyroux \& Moyano, 1987: 49; Desqueyroux-Faúndez, 1989: 121, pl. III, figs 18ac, pl. XII, figs 71; Barthel et al., 1990: 122; Pansini et al., 1994: 76; Gutt \& Koltun, 1995: 230; Desqueyroux-Faúndez \& Van Soest, 1996: 55, figs 105-110; Cattaneo-Vietti et al., 1999; Ríos et al., 2004: 107, text-figs 7A-J.

Tedania armata Sarà, 1978: 51, text-figs 30-31; Pansini \& Sarà, 1999: 205.

Further synonym see Koltun (1964) and DesQUeYRouX-Faúndez (1989).
Studied material. MCNPOR 3134, St. 5062, Elephant I.: $61^{\circ} 12^{\prime}$ S-554ㅇW, 98 m, 26.II.1987, PROANTAR V, "otter-trawl" coll.

Examined material for comparison. Tedania (Tedaniopsis) charcoti (Topsent, 1907), collected by the Brazilian Antarctic Program, locality: Joinville I., MCNPOR 1136 (identified by B. Mothes \& C. B. Lerner).

Description. (Fig. 58) Massive, amorphous specimen; dimensions in cm: 8.2 length, 4.9 width, 3.8 height; smooth surface, characterized by a thin membrane, which is absent in some regions, but some ridges and grooves occur over the surface; only one oscule was observed $(0.15 \mathrm{~cm}$ in diameter $)$. Preserved material with compressible consistency, colour beige.

Skeleton. Ectosome composed of a palisade of tornotes, sometimes forming discrete bouquets (Fig. 59). Choanosome with a confuse reticulation, with irregular tracts of styles, connected by isolated spicules (Fig. 60); spongin abundant. Onychaetes dispersed along the choanosome.

Spicules. Megascleres: styles - of varied curvature and/or sinuous (Fig. 61); apical extremity acerate or slightly mucronate (Fig. 62); tornotes - straight or slightly curved (Fig. 63); conical to acerate extremities, slightly swollen at the terminal portion (Fig. 64). Microscleras: onychaetes I - poorly developed spines (Fig. 65), sharp-pointed extremities (Fig. 66); onychaetes II well developed spines, thicker towards the basal extremity (Fig. 67); asymmetrical ends (Fig. 68). Measurements (Tab. VIII).

Remarks. The spicule measurements of our material are very similar to those previously reported for this species, with the exception of Desqueyroux-FaúndeZ (1989) and SARÀ (1978), who reported smaller styles. In regard to the onychaetes, DESQUEYROUX-FAúnDEZ (1989) and SARÀ (1978) cited only one category of these spicules, instead of two as found in the present study.

GAINo et al. (1994) reported a constant association of this species with diatoms, supporting the notion that diatoms may be responsible for the presence of chlorophyll pigments and thus suggesting the existence of a different trophic strategy linked to the Antarctic environment. CAPON et al. (1993) observed the occurrence of a high natural concentration of cadmium and zinc, with an extract being able to modulate protein phosphorylation and to inhibit the growth of several species of bacteria.

Distribution. Indian Ocean: Kerguelen I. (LévI 1964; BouRYEsnault \& Van Beveren 1982). South America: Chile (Desqueyroux 1976; Desqueyroux \& Moyano 1987; Desqueyroux-Faúndez \& Van Soest 1996); Argentina (Cuartas 1992); Magellan Strait (SARÀ 1991; PANSINI \& SARÀ 1999); Tierra del Fuego (SARÀ 1978); Falkland I. (Cuartas 1986); South Georgia; Shag Rocks (Burton 1932); Burdwood Bank (Topsent 1913; BurTon 1934). Antarctica: South Orkney I. (Burton 1940); Graham Land (Topsent 1907; 1908); Queen Mary Land; Adelie Land (BurTon 1938); Wilhelm II Land (Koltun 1964); Enderby Land; McRobertson Land (Koltun 1976); Victoria Land (Pansini et al. 1994; Cattaneo-Vietti et al. 1999); Weddell Sea (Barthel et al. 1990; Gutt \& Koltun 1995); South Shetland I.: Greenwich I. (Desqueyroux 1975); Livingston I. (Ríos

Revista Brasileira de Zoologia 24 (3): 742-770, setembro 2007 

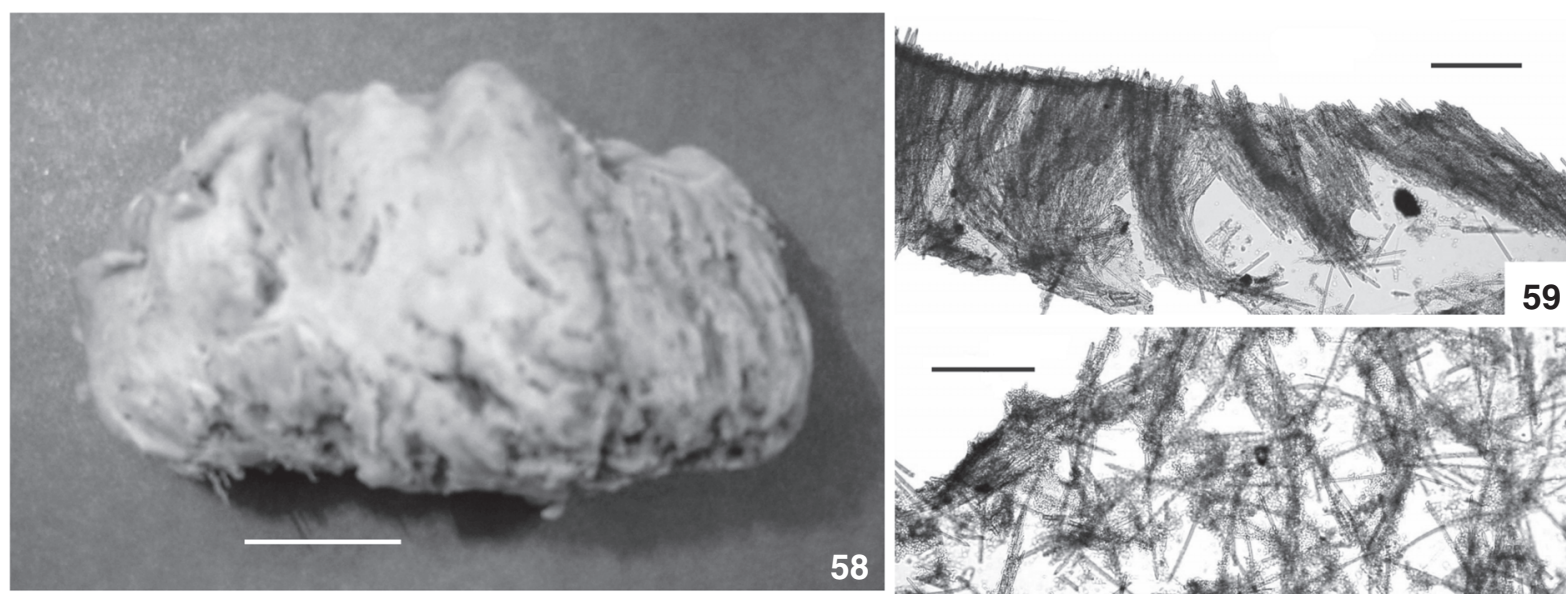

58

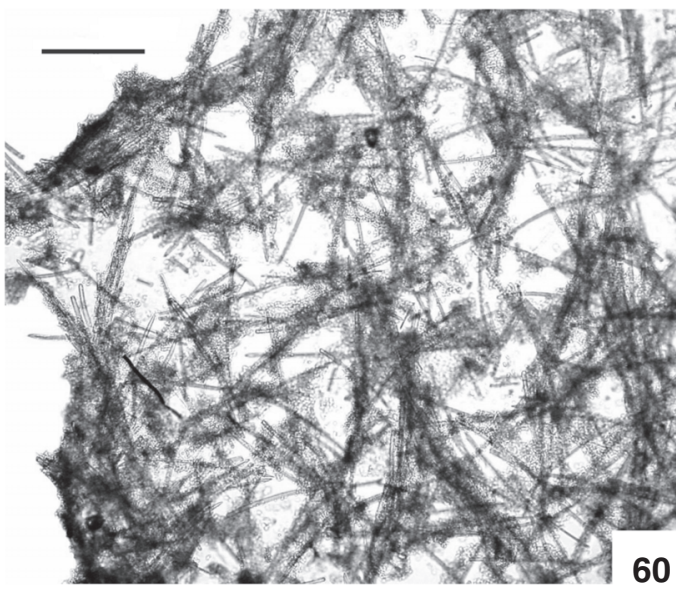

62

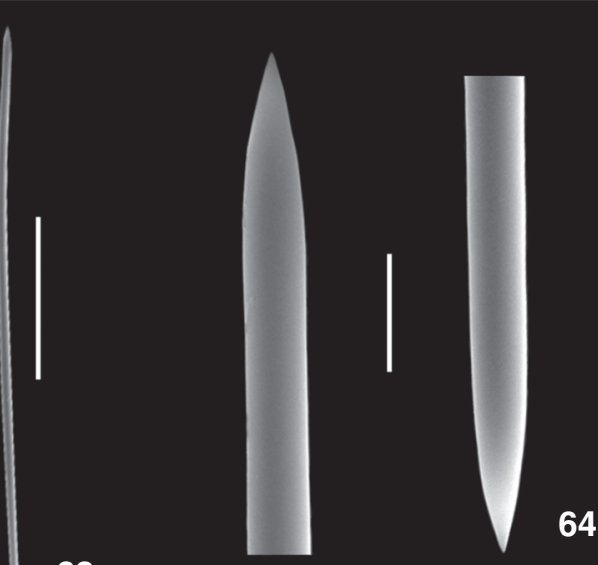

65

63
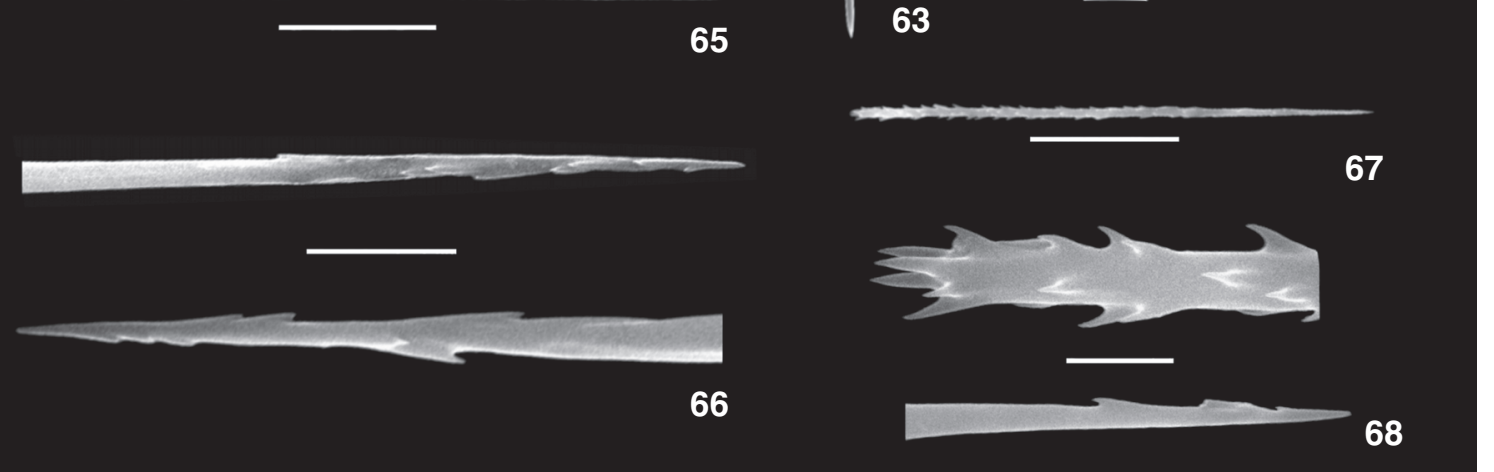

Figures 58-68. Tedania (Tedaniopsis) charcoti: (58) preserved specimen; (59) ectosomal arrangement of the skeleton; (60) choanosomal skeleton; (61) style; (62) detail of style extremities; (63) tornote; (64) detail of tornote extremities; (65) onychaete l; (66) detail of onychaete I extremities; (67) onychaete II; (68) detail of onychaete II extremities. Scale bars: (58) $2 \mathrm{~cm}$; (59) $300 \mu \mathrm{m}$; (60) 500 $\mu \mathrm{m}$; (61) $100 \mu \mathrm{m}$; (62) $15 \mu \mathrm{m}$; (63) $100 \mu \mathrm{m}$; (64) $10 \mu \mathrm{m}$; (65) $50 \mu \mathrm{m}$; (66) $3 \mu \mathrm{m}$; (67) $20 \mu \mathrm{m}$; (68) $2 \mu \mathrm{m}$. 
Table VIII. Tedania (Tedaniopsis) charcoti, spicules measurements.

\begin{tabular}{ccccc}
\hline MCNPOR & Styles & Tornotes & Onychaetes I & Onychaetes II \\
\hline 3134 & $380-457.2-520 / 10-12.8-15$ & $300-348.4-390 / 6.3-7.6-8.8$ & $200-228.6-310 / 1.0-1.8-2.5$ & $75-90.1-115 /<1.0$ \\
\hline
\end{tabular}

et al. 2004); Elephant I. (Desqueyroux-Faúndez 1989; present study). Bathymetry: 0 m (DesqueYroux-Faúndez 1989) to $728 \mathrm{~m}$ (BURTON 1932).

\section{Tedania (Tedaniopsis) vanhoffeni (Hentschel, 1914) Figs 69-78, Tab. IX}

Tedania vanhoffeni Hentschel, 1914: 90, pl. VI, fig. 13; Koltun, 1964: 60; Boury-Esnault \& Van Beveren, 1982: 96, pl. XVI, fig. 62, text-figs 27a-f; Barthel et al., 1990: 122; 1997: 48.

Studied material. MCNPOR 3108, St. 4871, Bransfield Strait: $63^{\circ} 16^{\prime} \mathrm{S}-59^{\circ} 55^{\prime} \mathrm{W}, 264 \mathrm{~m}$, 08.II.1986, PROANTAR IV, "beam-trawl" coll.

Examined material for comparison. Tedania (Tedaniopsis) vanhoffeni Hentschel 1914, collected by the Deutsche Südpolar Expedition, locality: Gauss Station, Wilhelm II Land, Antarctica, slide ZMB 4844 (holotype). Additional material: $T$. vanhoffeni, collected by the REVIZEE - Sul Program, locality: off Rio Grande do Sul State coast, Brazil, slide MCNPOR 3694.

Description. (Fig. 69) Fragment; dimensions in cm: 0.9 length, 0.7 width, 0.3 height; hispid surface, with protruding spicules; oscules and/or pores not observed. Preserved material (?) stiff consistency, colour beige.

Skeleton. (Fig. 70) Ectosome absent (broken). Choanosome composed of multispicular tracts, reinforced by a certain amount of spongin and several free spicules. Other details of the skeletal architecture were not analyzed due to the fragment degree of the sample.

Spicules. Megascleres: styles - straight, slightly curved or sinuous (Fig. 71); apical extremity blunt, some conical or stepped (Fig. 72); tornotes - straight (Fig. 73), unequal extremities (acerate and mucronate) (Fig. 74). Microscleres: onychaetes I - asymmetrical extremities; one of them acerate and microspined, the other a little more swelled with a well developed terminal spine (Figs 75, 76); onychaetes II (Figs 77-78) similar in morphology to onychaetes I, one extremity blunt with terminal spines and the other acerate with a larger spine. Measurements (Tab. IX).

Remarks. This is a species with few records; the present study provides a new occurrence of $T$. vanhoffeni. Illustration of spicules was improved by SEM analyses, mainly permitting detailed observation of the general morphology of the onychaetes.

Comparison with the holotype confirmed the identification of the sample (remeasurement: table IX). Subtle differences were noted in the holotype: thinner styles and onychaetes I, in addition to tornotes having swollen extremities with a slight microspination; on the other hand, both sets of material share certain characteristics, such as the sinuosity present in some styles, and mainly the details of the extremities of the two categories of onychaetes.

BARTHEL (1995) noted that individuals of this species are large and soft-bodied, and exude copious quantities of slime when disturbed. BARThel \& GutT (1992) observed that T. vanhoffeni is a very soft species, easily damaged in trawls.

Distribution. Indian Ocean: Kerguelen I. (Boury-Esnault \& Van Beveren 1982). South America: off Rio Grande do Sul State coast, Brazil (MотнEs et al. 2004b). Antarctica: Wilhelm II Land (Hentschel 1914; Koltun 1964); Weddell Sea (BARThel et al. 1990; 1997); Bransfield Strait (present study). Bathymetry: 46 m (Hentschel 1914) to 499 m (Koltun 1964).

\section{Tedania (Tedaniopsis) oxeata (Topsent, 1916) Figs 79-88, Tab. X}

Tedania oxeata Topsent, 1916: 169; Burton, 1938: 15; Vacelet \& Arnaud, 1972: 18; Desqueyroux, 1975: 65; Barthel et al., 1990: 122, 1997: 48; Gutt \& Koltun, 1995: 230; Mothes \& Lerner, 1995: 161, figs 33-41, 57.

Further synonym see DesqueYroux (1975).

Studied material. MCNPOR 1566, St. 4381, Bransfield Strait: $62^{\circ} 48^{\prime} \mathrm{S}-54^{\circ} 20^{\prime} \mathrm{W}, 280 \mathrm{~m}$, 18.I.1983, PROANTAR I, "beamtrawl" coll.; MCNPOR 2008, St. 4873, Bransfield Strait: 6325'S$62^{\circ} 05^{\prime} \mathrm{W}, 66$ m, 13.II.1986, PROANTAR IV, "beam-trawl" coll.

Examined material for comparison. Tedania (Tedaniopsis) oxeata (Topsent, 1916), collected by the Brazilian Antarctic Program, locality: Joinville I., MCNPOR 1134 (identified by B. Mothes \& C. B. Lerner).

Table IX. Tedania (Tedaniopsis) vanhoffeni, spicules measurements.

\begin{tabular}{ccccc}
\hline & Styles & Tornotes & Onychaetes I & Onychaetes II \\
\hline MCNPOR3108 & $590-649.6-720 / 26.3-32.9-40$ & $290-343.4-390 / 6.3-7.9-10$ & $389.5-440.9-513 / 3.5-4.7-5.8$ & $87.4-115.5-154.1 / 2.5$ \\
MCNPOR3694 & $418-471.4-532 / 16.3-20.3-23.8$ & $250-269-290 / 3.8-5.3-6.3$ & $290-338.2-370 / 2.5$ & $107.5-138.6-155 / 2.5-3.5-5.0$ \\
ZMB 3108 & $570-639-779 / 15-20.4-26.5$ & $275.5-329.7-361 / 4.6-5.8-6.9$ & $427.5-510.7-570 / 2.5$ & $66.7-90.1-110.4 / 2.5$ \\
\hline
\end{tabular}

Revista Brasileira de Zoologia 24 (3): 742-770, setembro 2007 

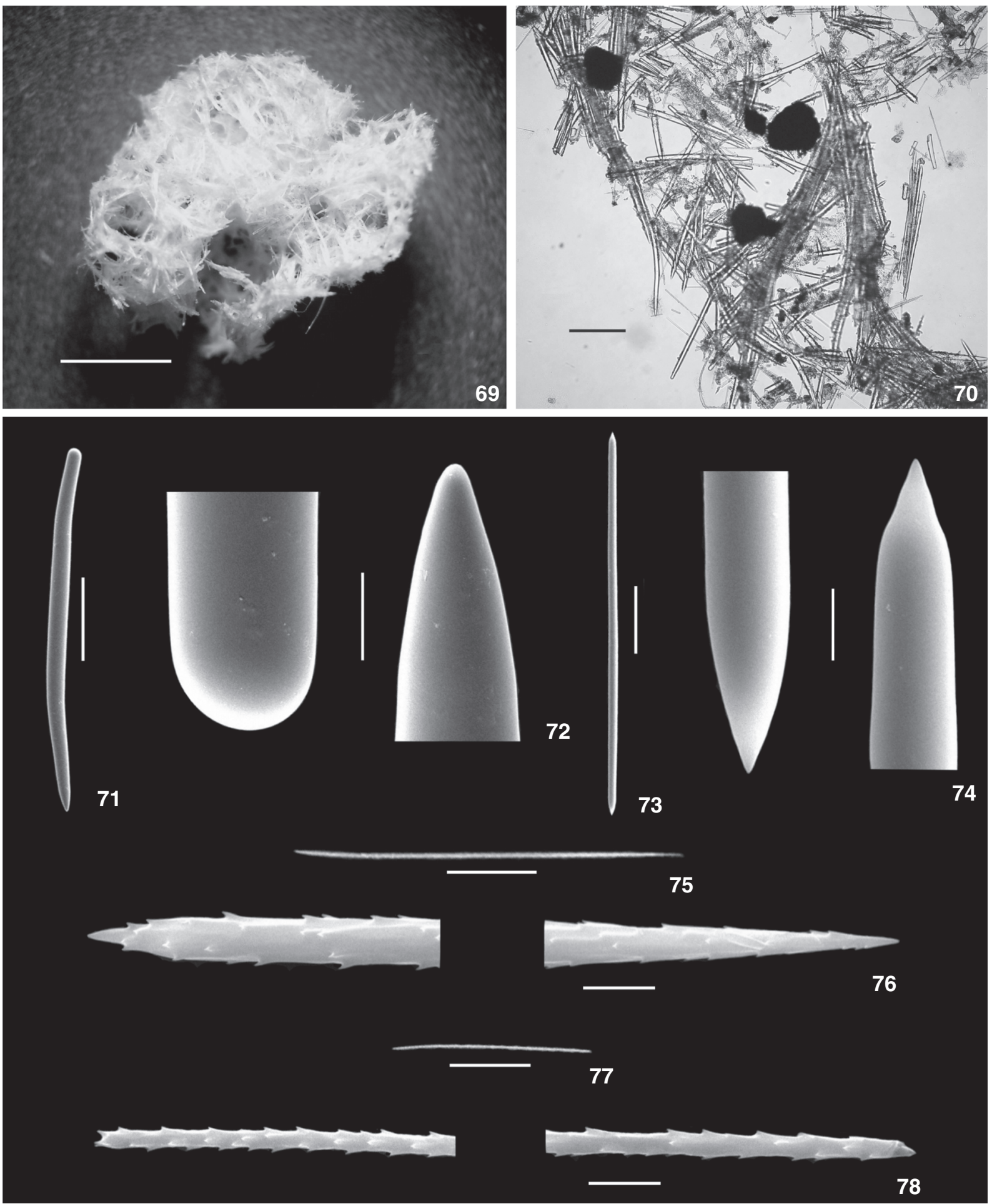

Figures 69-78. Tedania (Tedaniopsis) vanhoffeni: (69) preserved specimen; (70) skeleton; (71) style; (72) detail of style extremities; (73) tornote; (74) detail of tornote extremities; (75) onychaete I; (76) detail of onychaete I extremities; (77) onychaete II; (78) detail of onychaete II extremities. Scale bars: (69) $0.3 \mathrm{~cm}$; (70) $500 \mu \mathrm{m}$; (71) $150 \mu \mathrm{m}$; (72) $15 \mu \mathrm{m}$; (73) $50 \mu \mathrm{m}$; (74) $5 \mu \mathrm{m}$; (75) 100 $\mu \mathrm{m}$; (76) 5 $\mu \mathrm{m} ;(77) 50 \mu \mathrm{m} ;(78) 5 \mu \mathrm{m}$. 

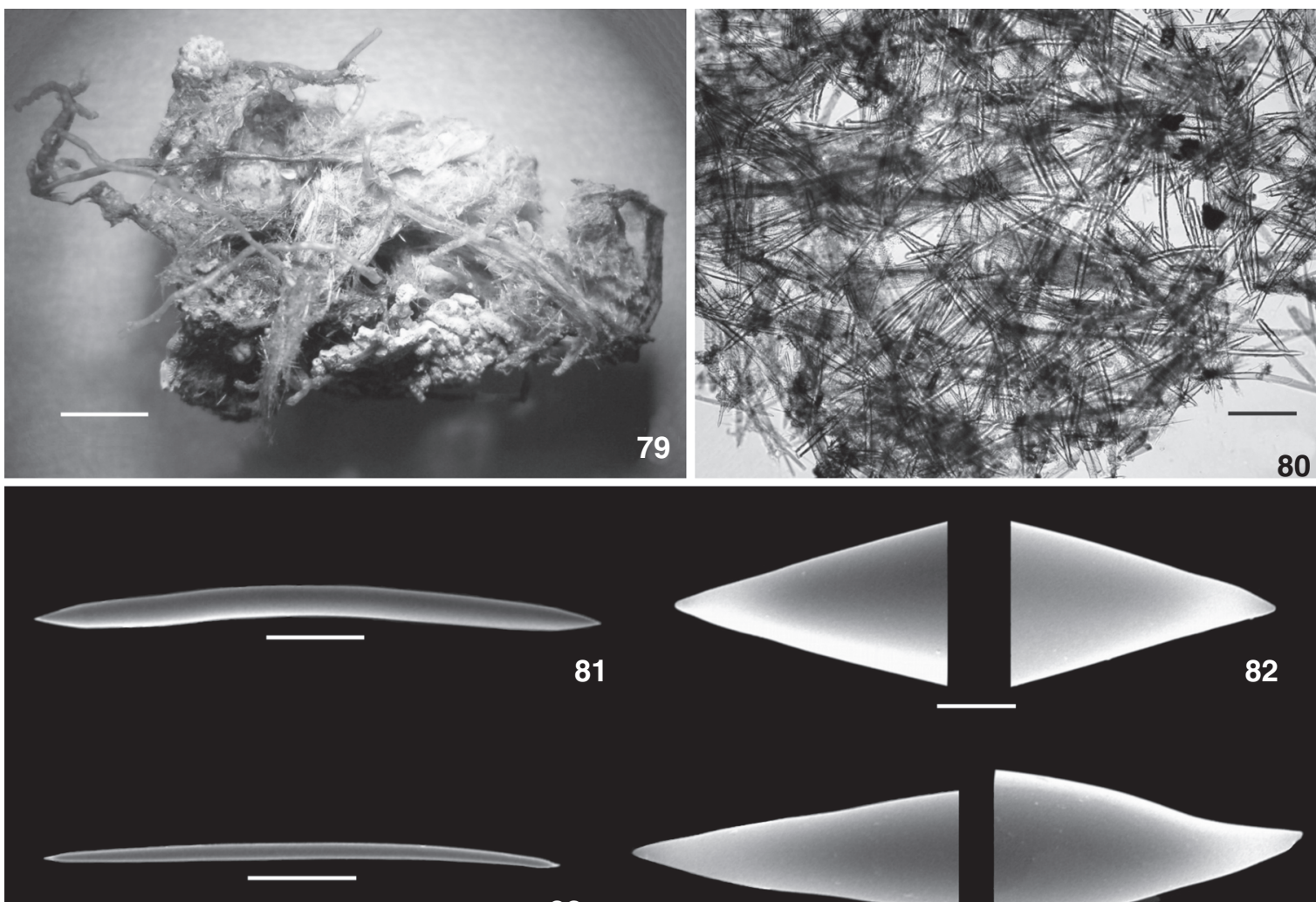

81

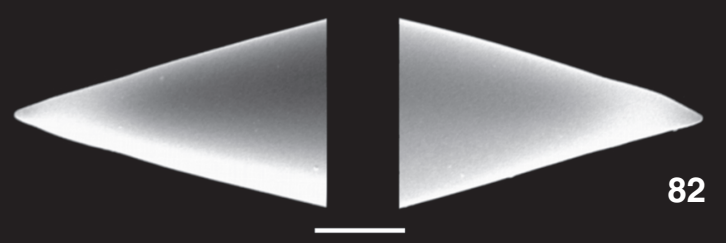

83
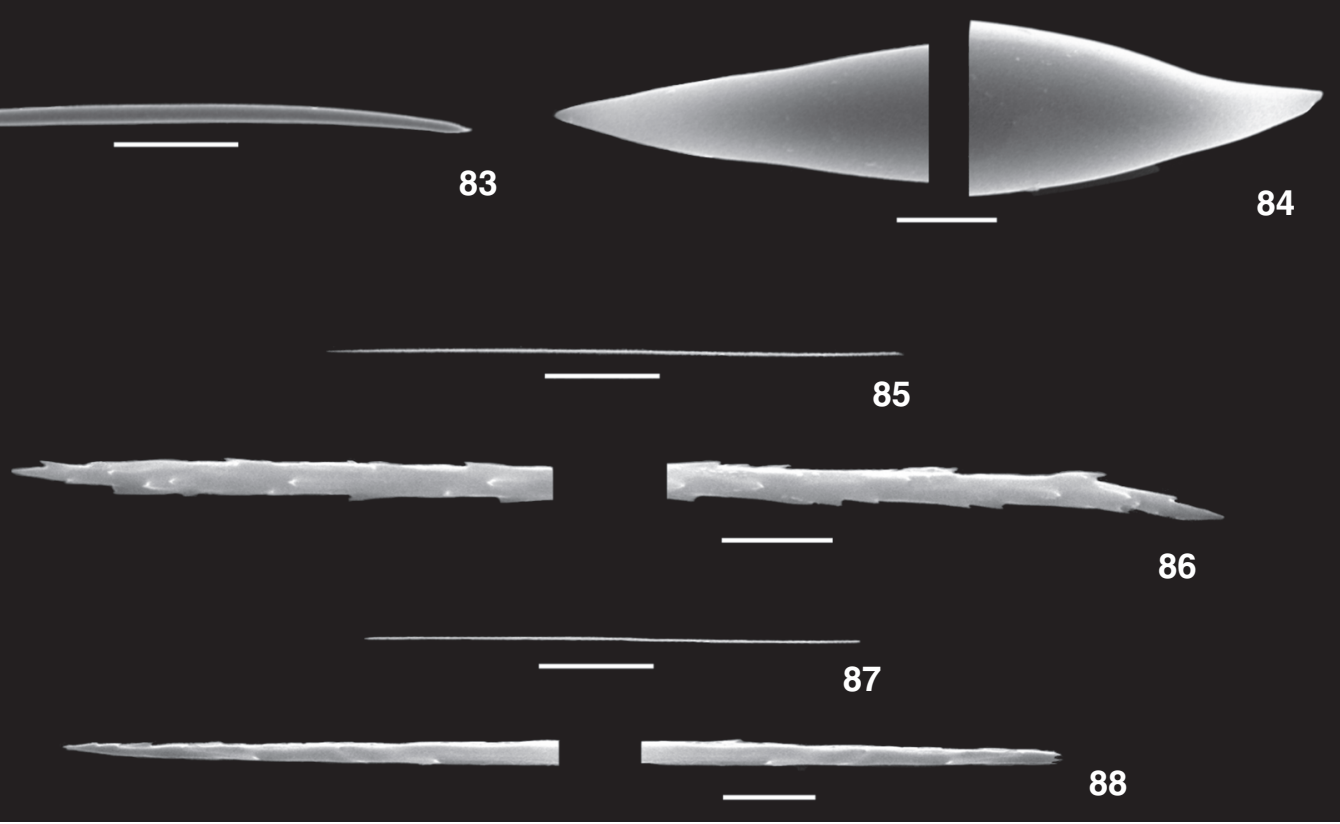

Figures 79-88. Tedania (Tedaniopsis) oxeata: (79) preserved specimen; (80) skeleton; (81) oxea; (82) detail of oxea extremities; (83) tornote; (84) detail of tornote extremities; (85) onychaete I; (86) detail of onychaete I extremities; (87) onychaete II; (88) detail of

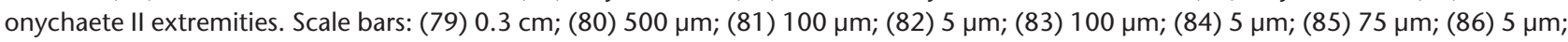
(87) $15 \mu \mathrm{m}$; (88) $5 \mu \mathrm{m}$.

Table X. Tedania (Tedaniopsis) oxeata, spicules measurements.

\begin{tabular}{ccccc}
\hline MCNPOR & Oxeas & Tornotes & Onychaetes I & Onychaetes II \\
\hline 1566 & $541.5-596.2-655.5 / 20.7-25.1-29.9$ & $380-458.9-532 / 6.9-11.7-16.1$ & $332.5-377.5-418$ & $55.2-71.5-92$ \\
2008 & $541.5-595.8-655.5 / 27.6-32.2-36.8$ & $418-477.1-541.5 / 11.5-15.3-17.3$ & $323-368.2-418$ & $59.8-71-98.9$ \\
\hline
\end{tabular}

Revista Brasileira de Zoologia 24 (3): 742-770, setembro 2007 
Description. MCNPOR 1566 (Fig. 79) Fragment infested with briozoans and small coralline plates; dimensions in $\mathrm{cm}$ : 1.7 length, 1.2 width, 0.7 height; due to their conservation state, it was not possible to characterize surface, consistency and presence of oscules. Preserved material fragile in consistency, colour beige.

Skeleton. (Fig. 80) Ectosome not visualized. Choanosome with a dense and confuse reticulation, and multispicular tracts; spongin present. In the rest of the skeleton the spicules are dispersed, sometimes grouped in small tufts.

Spicules. Megascleres: oxeas - straight or slightly curved (Fig. 81), some sinuous or twisted, and rarely style-like; extremities varying from conical to acerate, or slightly mucronate (Fig. 82); tornotes - straight or slightly curved (Fig. 83); extremities vary between conical, hastate, acerate or mucronate forms (Fig. 84). Microscleres: onychaetes I - with reduced but well distributed spines along the shaft (Fig. 85); acerate extremities, one of them bearing larger spines (Fig. 86); onychaetes II - well developed spines (Fig. 87); unequal extremities, one acerate and the other blunt with terminal spines (Fig. 88). Measurements (Tab. X).

Remarks. $T$. oxeata is very conspicuous in the subgenus Tedaniopsis, because it is until the present the only species of this group that has oxeas, rather than styles, in its spiculation. In regard to this, measurements provided by Topsent (1917) and Koltun (1964) indicated larger and thicker styles and tornotes. The material studied for the present report has spicules with similar dimensions to those reported by Mothes \& LeRner (1995).

Our samples are very fragmented, making it impossible to compare them in respect to external features. BARTHEL (1995) observed that $T$. oxeata has a hard, almost brittle consistency, in accordance with Topsent (1917), who described a sample of firm but fragile consistency. The present data extend the bathymetric distribution.

Distribution. Antarctica: Graham Land (Topsent 1916; 1917); Victoria Land (BurTon 1929); Queen Mary Land; Knox Land; Clarie Land; McRobertson Land; Banzare Land (KoltuN 1964); Adelie Land (VAcelet \& Arnaud 1972); Weddell Sea (Barthel et al. 1990; 1997; GuTt \& Koltun 1995); Joinville I. (MотнEs \& Lerner 1995); Bransfield Strait (present study); South Shetland I.: Greenwich I. (Desqueyroux 1975); Clarence I. (Burton 1932). Bathymetry: 66 m (present study) to 920 m (Koltun 1964).

\section{Mycalina Hajdu, Van Soest \& Hooper, 1994 Isodictyidae Dendy, 1924 Isodictya kerguelenensis (Ridley \& Dendy, 1886) Figs 89-93, Tab. XI}

Homoeodictya kerguelenensis Ridley \& Dendy, 1886: 346. Isodictya kerguelenensis; Lévi, 1956: 27, text-fig. 2-3; Koltun, 1976: 175; Vacelet \& Arnaud, 1972: 16; DesqueyrouxFaúndez, 1989: 113, pl. III, figs 11a-b, pl. IX, fig. 52; Barthel et al., 1990: 122; Cuartas, 1992: 75, text-figs 5, 6, 62; Ríos et al., 2004: 114, figs 14a-d.

Isodictya antarctica; Koltun, 1964: 42, pl. VIII, figs 17-18, 1976: 175; Desqueyroux, 1972: 51; Vacelet \& Arnaud, 1972: 16, figs 3-4; Barthel et al., 1990: 122.

Further synonym see Koltun (1964) and Desqueyroux-Faúndez (1989).

Studied material. MCNPOR 1952, St. 4862, Elephant I.: 61 ${ }^{\circ} 08^{\prime}$ S-54 $34^{\circ}$ 'W, 240 m, 01.II.1986, PROANTAR IV, “beamtrawl” coll.; MCNPOR 3122, St. 4874, Bransfield Strait: 63⒉5'S62 ${ }^{\circ} 19^{\prime} \mathrm{W}, 135$ m, 14.II.1986, PROANTAR IV, "beam-trawl" coll.

Examined material for comparison. Isodictya kerguelenensis (Ridley \& Dendy, 1886), collected by the Challenger Expedition, locality unknown, slide BMNH 1887.5.2.166a. Additional material: Isodictya antarctica (Kirkpatrick, 1908), collected by the Antarctic Discovery Expedition, locality unknown, slide BMNH 1928.ii.15.348a.

Description. (MCNPOR 1952) (Fig. 89) Massive, amorphous specimen; dimensions in $\mathrm{cm}$ : 11.8 length, 8.2 width, 1.8 height; conulose surface; oscules randomly scattered, at one side of the surface, in general at the apex of the conules, 0.1$0.4 \mathrm{~cm}$ diameter. Preserved material fragile but with incompressible consistency, colour beige.

Skeleton. (Fig. 90) Ectosome with no specialization. Choanosome formed by a dense and confuse reticulation, with multispicular tracts of oxeas (05-08 spicules, 100-150 $\mu \mathrm{m}$ thick) and a great amount of spongin and free spicules (including microscleres). Tracts tend to be thinner toward the surface.

Spicules. Megascleres: oxeas - straight to slightly curved (Fig. 91); acerate extremities (Fig. 92). Microscleres: isochelas (Fig. 93) - palmate, lateral alae well developed. Measurements (Tab. XI).

Table XI. Isodictya kerguelenensis, spicules measurements.

\begin{tabular}{ccc}
\hline MCNPOR & Oxeas & Isochelas \\
\hline 1952 & $400-479.4-560 / 22.5-27.4-32.5$ & $20-23.8-25$ \\
3122 & $550-658.2-770 / 15-21.6-30$ & $16.3-19.9-22.5$ \\
\hline
\end{tabular}

Remarks. Boury-Esnault \& VAn Beveren (1982) and Ríos et al. (2004) considered $I$. antarctica a junior synonym of $I$. kerguelenensis, which was previously noted by Koltun (1976). In the synonymy of $I$. antarctica are included two variations of I. kerguelenensis (simillima and cactoides), both proposed by HeNTSCHEL (1914).

DesqueYroux-FaúnDEZ (1989) stated that I. kerguelenensis is a polymorphic species, in regard to the external morphology and spicules. A large morphological difference can be observed in the form of the isochelas, mainly in the alae contour; Ríos et al. (2004) observed several different growth stages in these spicules. In the samples from the present study, a certain variation in the dimensions of the oxeas was noted, whereas the isochelas are identical. The comparative material corroborates 

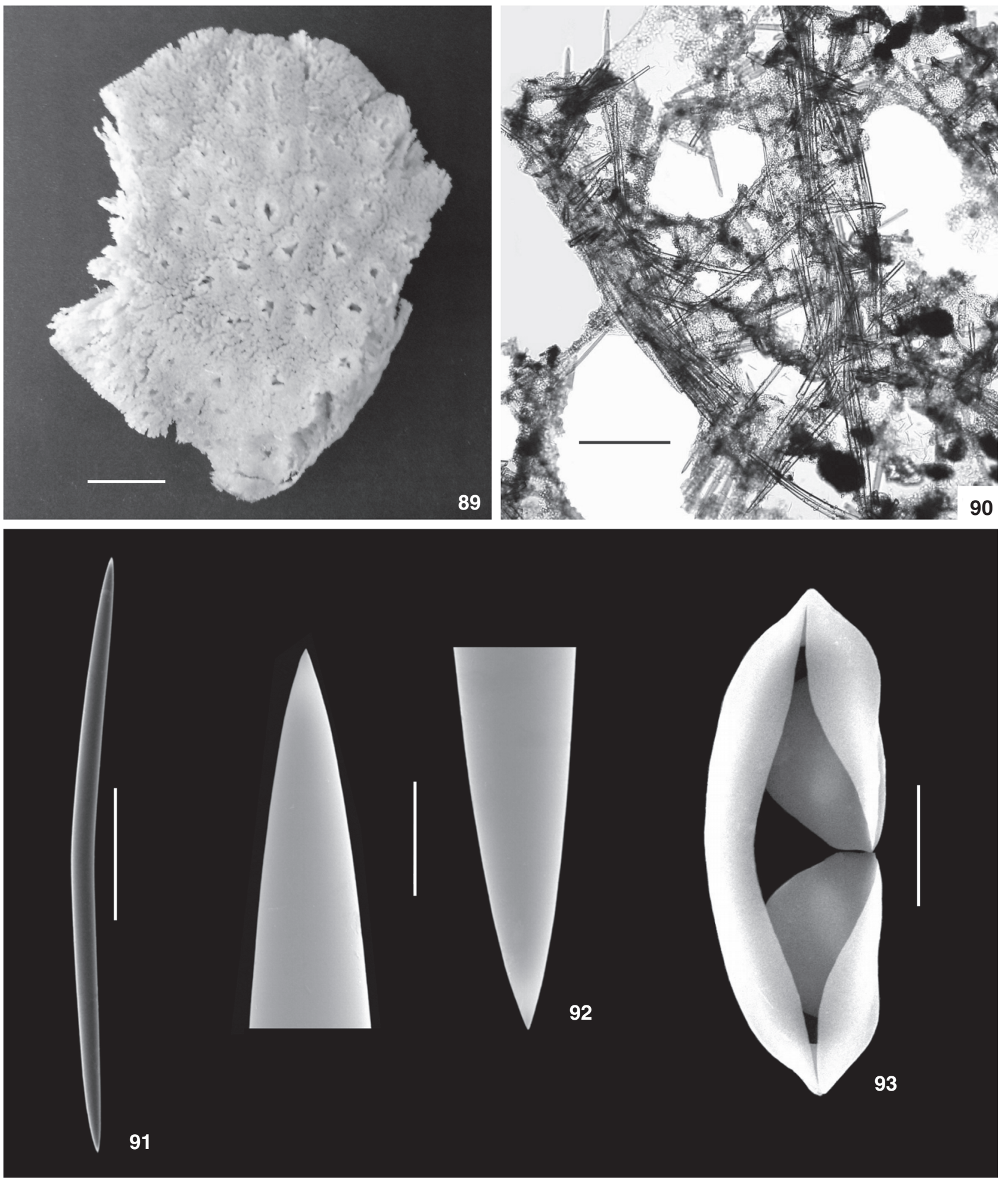

Figures 89-93. Isodictya kerguelenensis: (89) preserved specimen; (90) skeleton; (91) oxea; (92) detail of oxea extremities; (83) isochela. Scale bars: (89) $2 \mathrm{~cm}$; (90) $500 \mu \mathrm{m}$; (91) $150 \mu \mathrm{m}$; (92) $15 \mu \mathrm{m}$; (93) $5 \mu \mathrm{m}$.

Revista Brasileira de Zoologia 24 (3): 742-770, setembro 2007 
this information, and it was possible to observe the variations related to the species.

Distribution. Indian Ocean: Kerguelen I. (RIDley \& Dendy 1886; 1887; LÉVI 1956; Koltun 1964; Boury-EsNault \& VAN BeVEReN 1982); Heard I. (Koltun 1976; Boury-Esnault \& VAN Beveren 1982). South America: Argentina (Cuartas 1992); Falkland I.; South Georgia (Burton 1932). Antarctica: Graham Land (Topsent 1908; 1917); Victoria Land (KirKPATRICK 1908; BurTON 1929; 1938); Queen Mary Land (Burton 1938); Wilhelm II Land (Hentschel 1914); Adelie Land (Burton 1938; Vacelet \& Arnaud 1972); Queen Mary Land; Knox Land; Princess Elisabeth Land (Koltun 1964); Enderby Land; McRobertson Land (Koltun 1976); Weddell Sea (BARTHEL et al. 1990); South Shetland I.: Deception I. (Desqueyroux 1975); Livingston I. (Ríos et al. 2004); Greenwich I. (Desqueyroux 1972); Elephant I. (DesqueYroux-Faúndez 1989; present study); Bransfield Strait (DesqueYroux-FAúndez 1989; present study). Bathymetry: 2 $\mathrm{m}$ (Topsent 1908) to $1266 \mathrm{~m}$ (Koltun 1976).

\section{Isodictya Iankesteri (Kirkpatrick, 1907) Figs 94-98, Tab. XII}

Cercidochela lankesteri Kirkpatrick, 1907: 284; Burton, 1932: 287, 1934: 21; Desqueyroux, 1975: 61, pl. II, figs 21-22; Barthel et al., 1990: 122, 1997: 48; Gutt \& Koltun, 1995: 231.

Further synonym see Desqueyroux (1975).

Studied material. MCNPOR 3161, St. Ferraz, King George I.: $62^{\circ} 05^{\prime} \mathrm{S}-58^{\circ} 23^{\prime} \mathrm{W}, 20 \mathrm{~m}, 03 . \mathrm{II} .1985$, PROANTAR III, "scuba diving" coll.; MCNPOR 3133, 3137, 3142, St. 4743, Bransfield Strait: $62^{\circ} 30^{\prime} \mathrm{S}-54^{\circ} 16^{\prime} \mathrm{W}, 412 \mathrm{~m}, 28 . \mathrm{I} .1985$, PROANTAR III, "beam-trawl" coll.

Examined material for comparison. Isodictya lankesteri (Kirkpatrick, 1907), collected by the Antarctic Terra Nova Expedition, locality unknown, slide BMNH 1926.10.26.151a.

Description. (MCNPOR 3142) (Fig. 94) Massive, amorphous specimen; dimensions, in cm: 12.5 length, 5.0 width, 2.0 height; smooth surface, hispid to the touch; oscules in both the surfaces (larger $0.7 \mathrm{~cm}$ diameter), slightly elliptical. Under a magnifying glass it was observed, at the surface, the ends of spicule tracts; between them occur several rounded pores, 0.1-0.2 $\mathrm{cm}$ in diameter, randomly distributed along the surface. Preserved material extremely friable in consistency, colour white with marooned regions.

Skeleton. (Fig. 95) Ectosome with no specialization. Choanosome with longitudinal multispicular tracts of oxeas, 170$300 \mu \mathrm{m}$ thick, running toward the surface and responsible for the superficial hispidation. Between the tracts free oxeas and spongin also occur. Microscleres abundant along the choanosome.

Spicules. Megascleres: oxeas - slightly curved, some straight (Fig. 96), acerate extremities (Fig. 97). Microscleres: canonochelas (Fig. 98) - some with swollen central salience, alae can be slightly twisted, overlapped or even fused. Measurements (Tab. XII).

Remarks. The presence of a very conspicuous spicule (canonochela) allows I. lankesteri to be well differentiated among the species of Isodictya found in the study area.
In the present study, the description of the surface openings is extended, because BurTon $(1929 ; 1934)$ only cited the presence of openings in one of the sides of the sponge. Details of spicules and skeletal architecture are identical to the material examined for comparison. The bathymetric limit of the species is extended.

Distribution. South America: Shag Rocks (BURTON 1934). Antarctica: Victoria Land (KIRKPATRICK 1907; 1908; BurTON 1929); Palmer Archipelago (Burton 1932); Wilhelm II Land (Hentschel 1914); Knox Land (Koltun 1964); Enderby Land; McRobertson Land (Koltun 1976); Weddell Sea (Barthel et al. 1990; 1997; GutT \& Koltun 1995); Graham Land (Desqueyroux 1972); South Shetland I.: Greenwich I. (Desqueyroux 1975); King George I. (present study); Bransfield Strait (present study). Bathymetry: $20 \mathrm{~m}$ (present study) to $840 \mathrm{~m}$ (Koltun 1964).

Table XII. Isodictya lankesteri, spicules measurements.

\begin{tabular}{ccc}
\hline MCNPOR & Oxeas & Canonochelas \\
\hline 3133 & $280-382-490 / 15-21.1-27.5$ & $57.5-63.1-67.5$ \\
3137 & $270-374.8-500 / 15-23.2-35$ & $55-60.9-67.5$ \\
3142 & $300-396.6-520 / 12.5-22.5-30$ & $55-61.6-67.5$ \\
3161 & $290-385.2-520 / 17.5-23.6-30$ & $57.5-64.2-70$ \\
\hline
\end{tabular}

\section{Isodictya toxophila Burton, 1932 Figs 99-104, Tab. XIII}

Isodictya toxophila Burton, 1932: 286, pl. LII, figs 2-3, pl. LIII, figs 1-2, text-fig. 18; Koltun, 1964: 43, pl. VIII, figs 19-22; Barthel, et al. 1990: 122, 1997: 48; Gutt \& Koltun, 1995: 231.

Studied material. MCNPOR 2016, St. 4874, Bransfield Strait: $63^{\circ} 25^{\prime}$ S-62 ${ }^{\circ} 19^{\prime} \mathrm{W}, 135$ m, 14.II.1986, PROANTAR IV, "beamtrawl" coll.

Examined material for comparison. Isodictya toxophila Burton, 1932, collector unknown, locality: South Georgia, slide BMNH 1936.1.13.23a.

Description. (Fig. 99) Conical erect specimen, fixed to a rock fragment; dimensions in cm: 4.2 height, 1.9 width, 1.3 thickness; surface hispid to the touch, under magnifying glass conules with spicules protraction were observed; abundant oscules, $<0.1-0.4 \mathrm{~cm}$ diameter. Preserved material firm in consistency, colour greyish beige.

Skeleton. (Fig. 100) Ectosome with abundant spongin and exogenous material. Choanosome composed of coarse multispicular tracts, $180-400 \mu \mathrm{m}$ thick. Between the tracts spicules are irregularly distributed, in confusion.

Spicules. Megascleres: oxeas - slightly curved, rarely straight (Fig. 101), extremities varying from acerate to conical (Fig. 102). Microscleres: isochelas (Fig. 103) - palmate, alae with remarkably curved contour, overlapping the axis; toxas (Fig. $104)$ - reduced angle of opening, some almost straight. Measurements (Tab. XIII). 


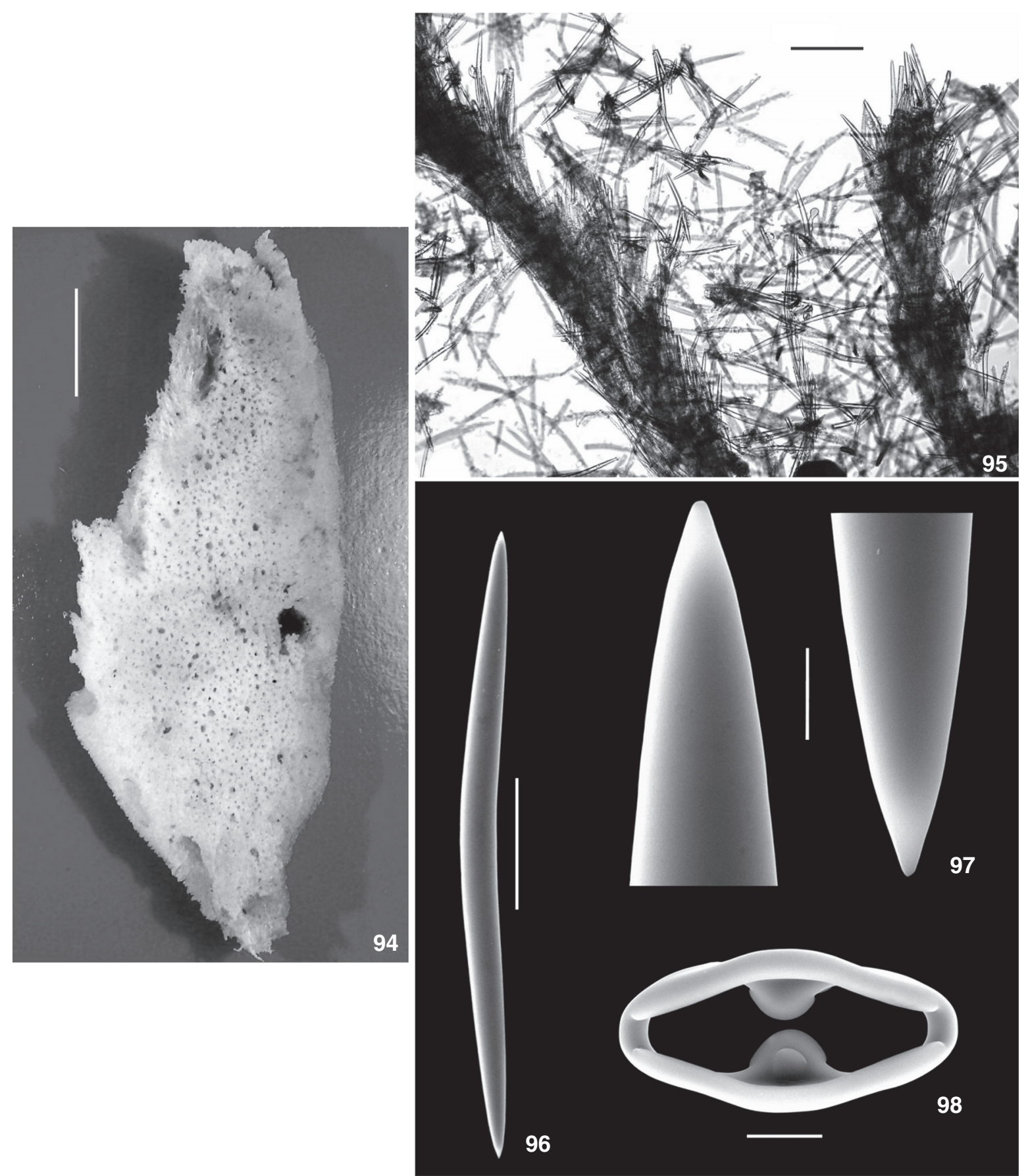

Figures 94-98. Isodictya lankesteri: (94) preserved specimen; (95) skeleton; (96) oxea; (97) detail of oxea extremities; (98) canonochelas. Scale bars: (94) $2 \mathrm{~cm}$; (95) $500 \mu \mathrm{m}$; (96) $75 \mu \mathrm{m}$; (97) $7 \mu \mathrm{m}$; (98) $15 \mu \mathrm{m}$.

Table XIII. Isodictya toxophila, spicules measurements.

\begin{tabular}{cccc}
\hline MCNPOR & Oxeas & Isochelas & Toxas \\
\hline \multirow{2}{*}{2016} & $\begin{array}{c}530-599.2-660 / \\
18.8-27.9-35\end{array}$ & $60-67.1-75$ & $170-207.3-237.5$ \\
\hline
\end{tabular}

Revista Brasileira de Zoologia 24 (3): 742-770, setembro 2007
Remarks. Up to the present, I. toxophila has a restricted geographic distribution, in South Georgia, South Shetland Is. and in some locations in the Weddell Sea, i.e., in an area with geographical continuity. 

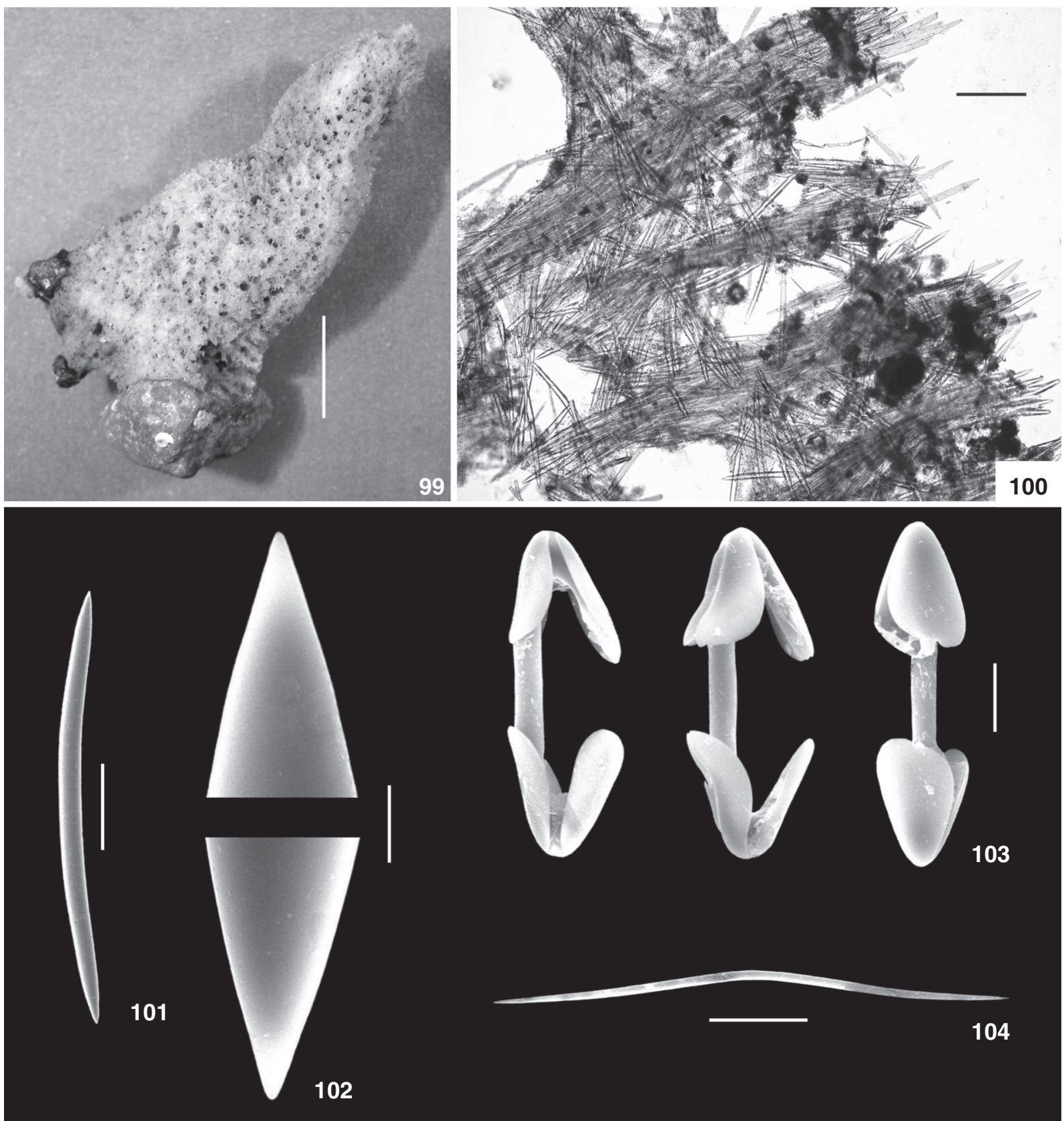

Figures 99-104. Isodictya toxophila: (99) preserved specimen; (100) skeleton; (101) oxea; (102) detail of oxea extremities; (103) isochelas; (104) toxas. Scale bars: (99) $1 \mathrm{~cm}$; (100) $500 \mu \mathrm{m}$; (101) $100 \mu \mathrm{m}$; (102) $5 \mu \mathrm{m}$; (103) $10 \mu \mathrm{m}$; (104) $40 \mu \mathrm{m}$.

In the original diagnosis of the species, BurTon (1932) supplied only the spicule dimensions, illustrating these in a simple form. Therefore, the present record enables us to provide better information on the spicules and consequently to improve the species diagnosis through SEM microphotographs.

Our sample is similar to that described by BurTon (1932), as well as in the skeletal architecture. The spicules concord well with descriptions and measurements provided by BURTON (1932) and Koltun (1964). The occurrence of toxas was not constant; Burton (1932) reported that their presence can vary, and they may even be rare or absent.

Distribution. South America: South Georgia (BUrTon 1932). Antarctica: Palmer Archipelago (BurTon 1932); Weddell Sea (Barthel et al. 1990; 1997; Gutt \& Koltun 1995); South Sh- 
etland I.: King George I. (Koltun 1964); Bransfield Strait (BuRTON 1932; present study). Bathymetry: 93 m (BURTON 1932) to $661 \mathrm{~m}$ (GutT \& Koltun 1995).

\section{Isodictya bentarti Ríos, Cristobo \& Urgorri, 2004 Figs 105-109, Tab. XIV}

Isodictya bentarti Ríos et al., 2004: 111.

Studied material. MCNPOR 1942, 1945, 1953, St. 4873, Bransfield Strait: $63^{\circ} 25^{\prime} \mathrm{S}-62^{\circ} 05^{\prime} \mathrm{W}, 66$ m, 13.II.1986, PROANTAR IV, "beam-trawl" coll.

Description. (MCNPOR 1945) (Fig. 105) Flabellate specimen; dimensions, in cm: 15 length, 10 width, 1.8 thickness; surface hispid to the touch; oscules aligned on the edges (0.1$0.6 \mathrm{~cm}$ diameter, equidistant $0.3-0.6 \mathrm{~cm}$ ), elliptical contour. Dermis is broken in some areas; in one of the sides small proeminences can be observed. Preserved material of very compressible consistency, colour beige, darker internally.

Skeleton. (Fig. 106) Ectosome formed by the terminal portion of the tracts, organized in discrete brushes which causes the superficial hispidation. Choanosome formed by a dense reticulation, composed of longitudinal multispicular tracts. Between the tracts oxeas and isochelas are present, together with certain amount of spongin, in an arrangement with no clear orientation.

Spicules. Megascleres: oxeas - slightly curved, some straight (Fig. 107), acerate extremities (Fig. 108). Microscleres: isochelas (Fig. 109) - palmate, lateral alae well developed; at the terminal portion of each alae there is a rounded protuberance. Measurements (Tab. XIV).

Table XIV. Isodictya bentarti, spicules measurements.

\begin{tabular}{ccc}
\hline MCNPOR & Oxeas & Isochelas \\
\hline 1942 & $370-481.6-580 / 17.5-24.3-33.8$ & $55-66.9-77.5$ \\
1945 & $380-472-550 / 15-26-32.5$ & $57.5-65.1-70$ \\
1953 & $370-471.8-560 / 17.5-26-32.5$ & $55-66.4-77.5$ \\
\hline
\end{tabular}

Remarks. The species is endemic to the South Shetland Is.: it was originally described from Livingston Is., and is now confirmed from the Bransfield Strait near Low Is., which belongs to South Shetland Is. The bathymetric distribution of the species is extended.

Both the characteristics of the external morphology and the details of the spicules reported in the original description (Ríos et al., 2004) are here corroborated.
According to Ríos et al. (2004), another sympatric species of Isodictya which shows similar characteristics to $I$. bentarti is I. grandis (Ridley \& Dendy, 1886). However, the latter has a different oscular pattern, besides differing in the morphology of the oxeas and isochelas.

Distribution. Antarctica: South Shetland I.: Livingston I. (Ríos et al. 2004); Bransfield Strait (present study). Bathymetry: $24 \mathrm{~m}$ (Ríos et al. 2004) to $66 \mathrm{~m}$ (present study).

\section{Latrunculina Kelly \& Samaai, 2002 Latrunculiidae Topsent, 1922}

\section{Latrunculia (Latrunculia) brevis Ridley \& Dendy, 1886} Figs 110-115, Tab. XV

Latrunculia brevis Ridley \& Dendy, 1886: 492; Topsent, 1915: 40, text-fig. 5

Further synonym see SAMAaI et al. (2006).

Studied material. MCNPOR 1985, St. 4861, Elephant I.: $61^{\circ} 02^{\prime} \mathrm{S}-54^{\circ} 55^{\prime} \mathrm{W}, 362 \mathrm{~m}, 01 . \mathrm{II} .1986$, PROANTAR IV, “beamtrawl" coll.

Examined material for comparison. Latrunculia (Latrunculia) brevis Ridley \& Dendy, 1886, collected by the Challenger Expedition, locality: off Rio de la Plata, Argentina, slide BMNH 1887.5.2.269 (holotype).

Description. (Fig. 110) Massive, amorphous specimen, dimensions in cm: 6.9 length, 3.5 width, 1.3 thickness; surface densely areolated, porefields measuring $0.2-0.8 \mathrm{~cm}$ diameter; some areas with smooth surface, with a slight spicule protraction. Oscular openings are visible inside the porefields (0.1-0.2 $\mathrm{cm}$ in diameter). Preserved material extremely fragile in consistency, colour dark brown.

Skeleton. (Fig. 111) Ectosome formed by a palisade of microscleres and a certain amount of spongin. Subectosomal region composed of a compact layer of megascleres in confusion. Choanosome bearing a dense arrangement of megascleres, disposed in irregular multispicular tracts and forming irregular meshes. Free spicules were also observed.

Spicules. Megascleres: anisostyles - smooth, straight to sinuous (Fig. 112); apical extremity varying from conical to acerate (Fig. 113). Microscleres: anisodiscorhabds (Fig. 114) - between manubrium and median whorl the shaft is slightly swollen. Imature spicules also occur (Fig. 115). Measurements (Tab. XV).

Remarks. SAMAai et al. (2006) made a complete revision of the genus Latrunculia, designating the correct identifications for $L$. brevis and its respective synonymy, and giving the geographical and bathymetric distribution of the species.

Table XV. Latrunculia (Latrunculia) brevis, spicules measurements.

\begin{tabular}{|c|c|c|c|c|c|c|c|}
\hline \multirow{2}{*}{ MCNPOR } & \multirow{2}{*}{ Anisostyles } & \multicolumn{6}{|c|}{ Anisodiscorhabds } \\
\hline & & Total lenght & Manubrium & Shaft & Median whorl & Subsidiary whorl & Apical whorl \\
\hline 1985 & $\begin{array}{c}427.5-475-503.5 / \\
6.9-10.3-11.5\end{array}$ & $50.6-60-64.4$ & $13.8-18.7-23$ & $\begin{array}{c}16.1-18-20.7 / \\
4.6-5.7-6.9\end{array}$ & $27.6-32.7-36.8$ & $24.2-29-32.2$ & $16.1-21.2-23$ \\
\hline
\end{tabular}



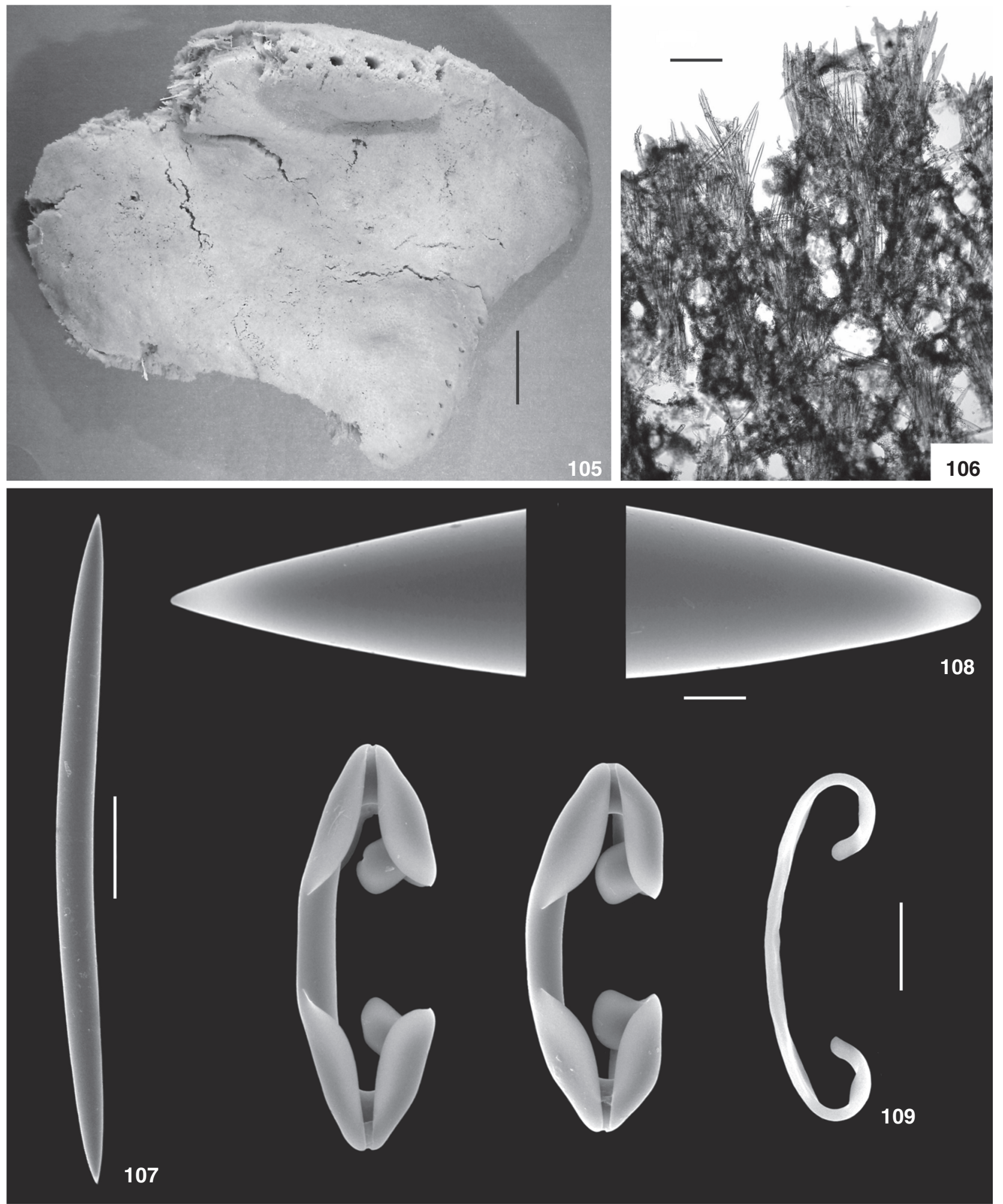

Figures 105-109. Isodictya bentarti: (105) preserved specimen; (106) skeleton; (107) oxea; (108) detail of oxea extremities; (109) isochelas. Scale bars: (105) $2 \mathrm{~cm}$; (106) $500 \mu \mathrm{m}$; (107) $75 \mu \mathrm{m}$; (108) $5 \mu \mathrm{m}$; (109) $15 \mu \mathrm{m}$. 


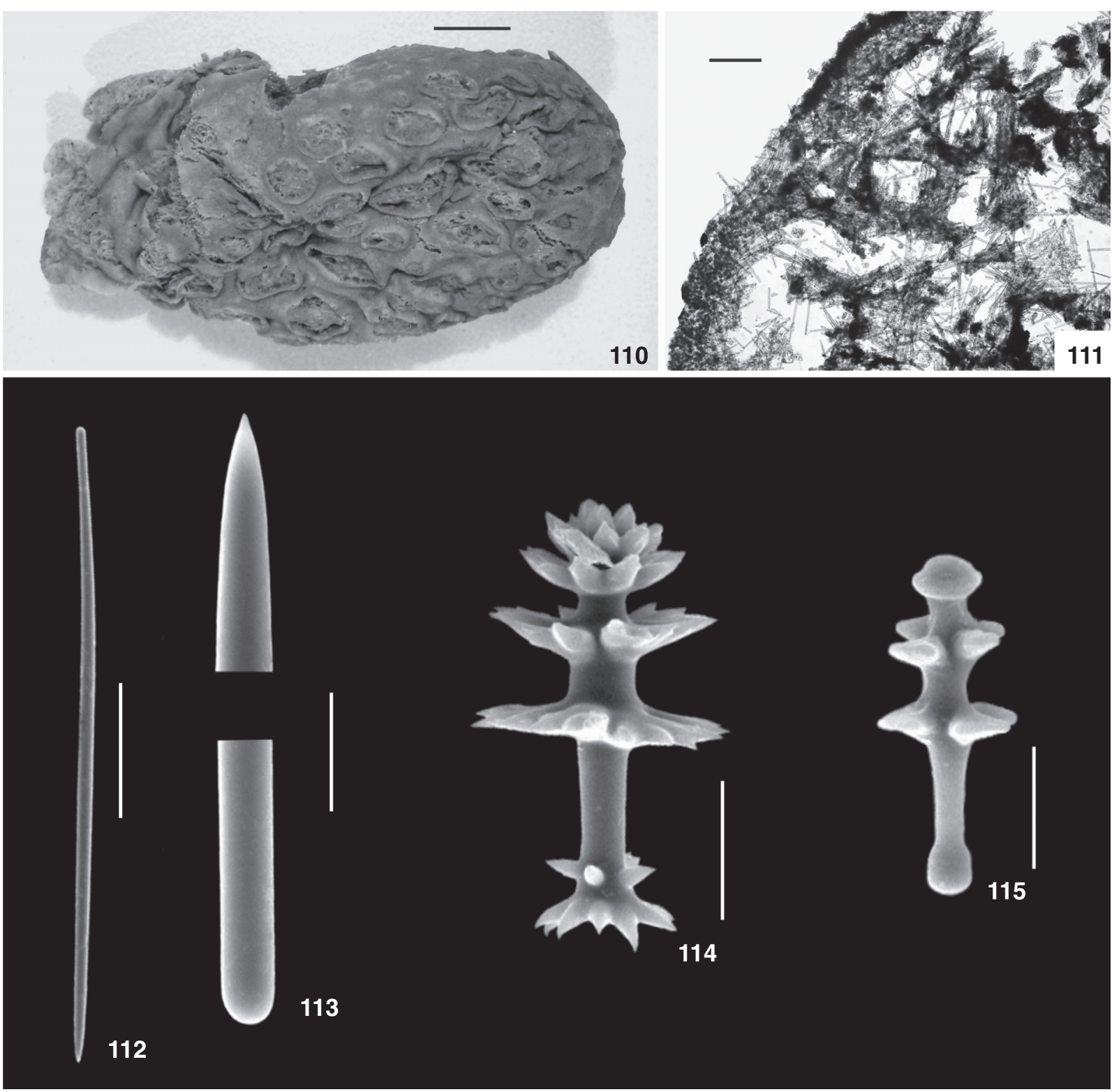

Figures 110-115. Latrunculia (Latrunculia) brevis: (110) preserved specimen; (111) skeleton; (112) anisostyle; (113) detail of anisostyle extremities; (114) anisodiscorhabd; (115) immature anisodiscorhabd. Scale bars: (110) $1 \mathrm{~cm}$; (111) $500 \mu \mathrm{m}$; (112) $100 \mu \mathrm{m}$; (113) 15 $\mu \mathrm{m}$; (114) $20 \mu \mathrm{m}$; (115) $15 \mu \mathrm{m}$.

The descriptions of some features, such as the external morphology, details of the skeleton and microscleres given by SAMAAI et al. (2006) concord with our material, which is quite similar to the holotype.

Distribution. South America: Uruguay (BuRTon 1940); Argentina (Ridley \& Dendy 1886; 1887); Falkland I. (Burton 1932); Burdwood Bank (Topsent 1915). Antarctica: SamaAi et al. (2006) (unknown locality); South Shetland I.: Elephant I. (present study). Bathymetry: $46 \mathrm{~m}$ to $1500 \mathrm{~m}$ (SAMAAI et al. 2006).

\section{Latrunculia (Latrunculia) biformis (Kirkpatrick, 1908)}

Figs 116-121, Tab. XVI

Latrunculia apicalis var. biformis Kirkpatrick, 1908: 14, pl. XV, figs $1-7$.

Latrunculia biformis; Pansini et al., 1994: 69; Cattaneo-Vietti et al., 1999: 540; Ríos et al., 2004: 117, text-fig. 15A-J.

Further synonym see Samai et al. (2006).

Studied material. MCNPOR 3128, St. 'D', King George I.:

Revista Brasileira de Zoologia 24 (3): 742-770, setembro 2007 

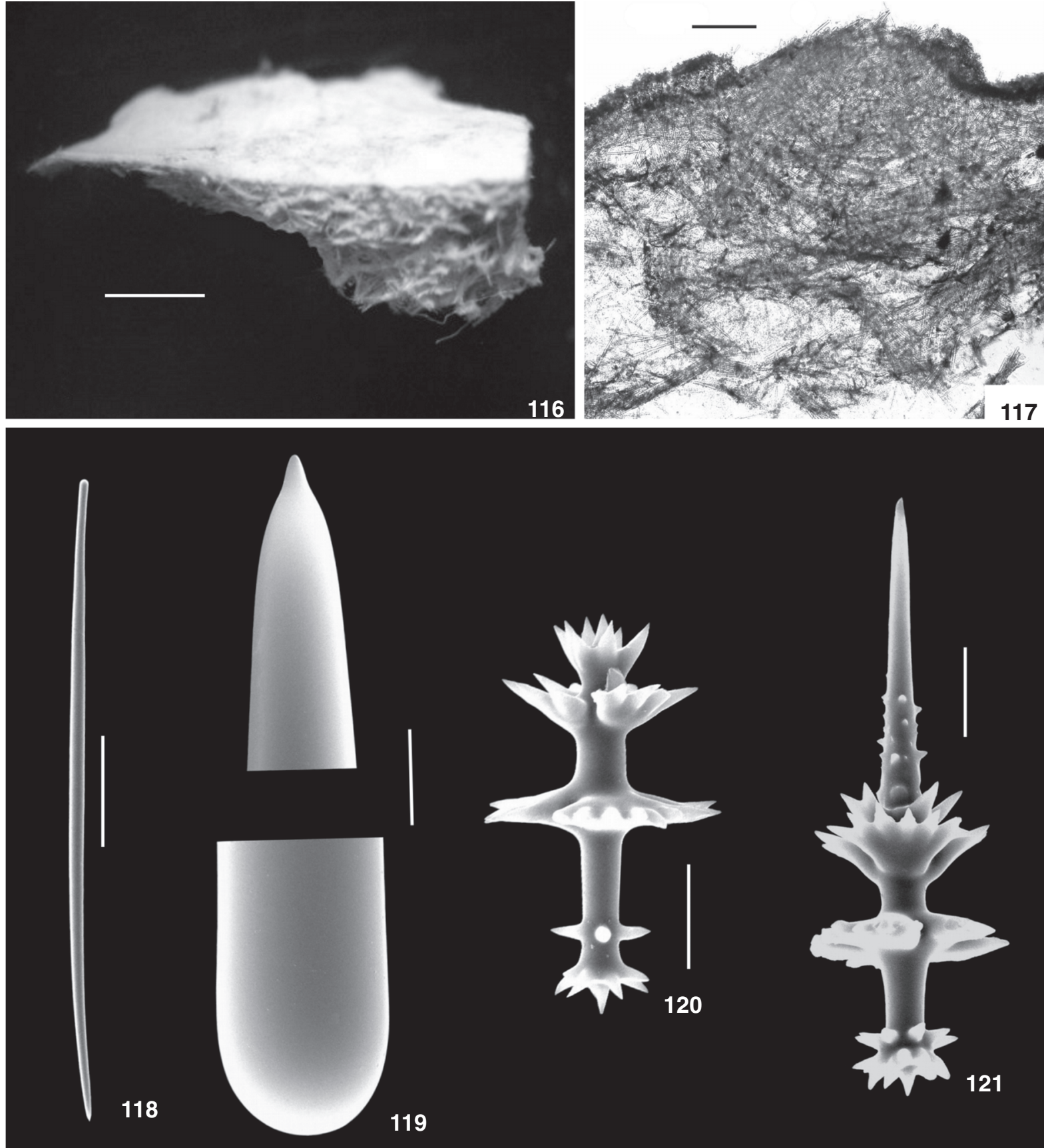

.

Figures 116-121. Latrunculia (Latrunculia) biformis: (116) preserved specimen; (117) skeleton; (118) anisostyle; (119) detail of anisostyle extremities; (120) anisodiscorhabd; (121) Aciculodiscorhabd. Scale bars: (116) $0.2 \mathrm{~cm}$; (117) $500 \mu \mathrm{m}$; (118) $100 \mu \mathrm{m}$; (119) $5 \mu \mathrm{m}$; (120), (121) $20 \mu \mathrm{m}$.

$62^{\circ} 05^{\prime}$ S-58 $23^{\prime} \mathrm{W}, 25 \mathrm{~m}, 05 . \mathrm{I} .1990$, PROANTAR VIII, “scuba diving" coll.

Examined material for comparison. Latrunculia (Latrunculia) biformis (Kirkpatrick, 1908), collected by the Ant- arctic Terra Nova Expedition, locality unknown, slide BMNH 1910.26.154a.

Description. (Fig. 116) Small fragment, dimensions in cm: 0.9 length, 0.6 width, 0.4 thickness; smooth surface, oscules

Revista Brasileira de Zoologia 24 (3): 742-770, setembro 2007 
Table XVI. Latrunculia (Latrunculia) brevis, spicules measurements.

\begin{tabular}{|c|c|c|c|c|c|c|c|}
\hline \multirow{2}{*}{ MCNPOR } & \multirow{2}{*}{ Anisostyles } & \multicolumn{6}{|c|}{ Anisodiscorhabds } \\
\hline & & Total lenght & Manubrium & Shaft & Median whorl & Subsidiary whorl & Apical whorl \\
\hline 3128 & $\begin{array}{c}484.5-541.9-579.5 / \\
10.4-12.3-13.8\end{array}$ & $\begin{array}{c}62.1-67.1-78- \\
.2 \\
\end{array}$ & $16.1-18.8-23$ & $\begin{array}{c}15-17.8-21.9 / \\
6.9-7.3-8.1\end{array}$ & $35.7-40.9-46$ & 29.9-33.9-39.1 & $16.1-19.4-23$ \\
\hline \multirow[b]{2}{*}{ MCNPOR } & \multicolumn{7}{|c|}{ Aciculodiscorhabds } \\
\hline & Total lenght & Manubrium & Shaft & Median whorl & Subsidiary whorl & Apical whorl & $\begin{array}{c}\text { Apical } \\
\text { projection }\end{array}$ \\
\hline 3128 & $105.8-120-133.4$ & $17.3-21-25.3$ & $\begin{array}{c}20.7-23.4-26.5 / \\
6.9-7.4-9.2\end{array}$ & $36.8-41.6-46$ & $29.9-35.8-39.1$ & $18.4-22.4-25.3$ & $\begin{array}{c}39.1-51.2-64.4 / \\
5.8-6.8-8.1\end{array}$ \\
\hline
\end{tabular}

not observed. Preserved material compressible but fragile consistency, colour light brown at the surface, being darker internally.

Skeleton. (Fig. 117) Characteristics of the ectosome, choanosome and subectosomal region identical to the description cited for $L$. brevis.

Spicules. Megascleres: anisostyles - smooth, straight to sinuous (Fig. 118); apical extremity conical/acerate (Fig. 119). Microscleres: anisodiscorhabds (Fig. 120) - presenting the same characteristics observed in L. brevis. Aciculodiscorhabds (Fig. 121) - in general very similar to anisodiscorhabds, only differing in having a well developed spined apical projection. Measurements (Tab. XVI).

Remarks. Because SAMAAI et al. (2006) made a complete revision of the genus, it is unnecessary to comment on the synonymy of the species and the corresponding identifications.

It was not possible to compare our sample to the external morphology described by SAMAAI et al. (2006), because the specimen is not entire. Other morphological features of the species, such as the skeletal patterns and spicules, concord with the specimen studied. Nevertheless, this species is considered to show a wide variation in the dimensions and structure of the discorhabds. The existence of such variation was confirmed by analysis of comparative material, in which were observed aciculodiscorhabds with a reduced apical projection.

Currently, the following species are valid for the Antarctic Faunistic Complex: L. bocagei, L. apicalis, L. biformis, L. basilis and $L$. brevis. Probably the morphology of the discorhabds is the character that best distinguishes these species.

Distribution. Indian Ocean: Kerguelen I. (Boury-Esnault \& Van Beveren 1982). Africa: South Africa (SAmaAi et al. 2003). South America: Argentina (Ridley \& Dendy 1886; 1887). Antarctica: Victoria Land (KirKPATRICK 1908; Burton 1929; PANSInI et al. 1994; Cattaneo-Vietti et al. 1999); Princess Astrid Land; Queen Mary Land (Koltun 1964); South Shetland I.: Livingston I. (Ríos et al. 2004); King George I. (present study). Bathymetry: $18 \mathrm{~m}$ (Samail et al. 2003) to $1097 \mathrm{~m}$ (Ridley \& Dendy 1886).

\section{ACKNOWLEDGEMENTS}

We thank Clare Valentine (NHM), Alexander Plotkin (ZIN) and Carsten Lüter (ZMB) for the loan of comparative material; Eduardo Hajdu (Museu Nacional, Universidade Federal do Rio de Janeiro, Brazil) and Ruth Desqueyroux-Faúndez (Museum d'Histoire Naturelle de Genève, Geneva, Switzerland) for help in bibliography. We also thank CAPES and CNPq (Brazil) for research grants.

\section{REFERENCES}

BARTHEL, D. 1995. Tissue composition of sponges from the Weddell Sea, Antarctica: not much meat on the bones. Marine Ecology Progress Series 123: 149-153.

BARThEL, D. \& J. GuTt. 1992. Sponge associations in the eastern Weddell Sea. Antarctic Science 4 (2): 137-150.

Barthel, D.; O. Tendal \& K. PAnZer. 1990. Ecology and taxonomy of sponges in the eastern Weddell Sea shelf and slope communities. Berichte zur Polarforschung 68: 120-130.

Barthel, D.; O. Tendal \& S. Gatti. 1997. The Sponge Fauna of the Weddell Sea and Its Integration in Benthic Processes. Berichte zur Polarforschung 249: 44-52.

Boury-Esnault, N. \& M. Van Beveren. 1982. Les Démosponges du Plateau Continental de Kerguelen-Heard. Comité National Français des Rechearches Antarctiques 52: 1-175.

Burton, M. 1929. Porifera. Part II. Antarctic Sponges. National History Reports of the British Antarctic "Terra Nova" Expedition, 1910, Zoology 6 (4): 393-458.

Burton, M. 1932. Sponges. Discovery Reports 6: 237-392.

Burton, M. 1934. Sponges. Further Zoological Results of the Swedish Antarctic Expedition 1901-1903 3 (2): 1-58.

Burton, M. 1938. Non-calcareous sponges. Australasian Antarctic Expedition 1911-1914, Scientific Reports, Series C, Zoology and Botany, 9 (5): 3-22.

Burton, M. 1940. Las esponjas marinas del Museo Argentino de Ciencias Naturales. Anales del Museo Argentino de Ciencias Naturales "Bernardino Rivadavia" 40: 95-121.

Calcinai, B. \& M. Pansini. 2000. Four new demosponge species from Terra Nova Bay (Ross Sea, Antarctica). Zoosystema 22 (2): 369-381.

Capon, R.J.; K. Elsbury; M.S. Butler; C.C. Lu; J.N.A. Hooper; J.A.P. Rostas; J.K. O’Brien; M. Mudge \& A.T.R. Sim. 1993. Extraordinary levels of cadmium and zinc in a marine sponge, Tedania charcoti Topsent: inorganic chemical defense agent. Experientia 49: 263-264. 
Cattaneo-Vietti, R.; G. Bavestrello; C. Cerrano; E. Gaino; L. Mazzella; M. PansinI \& M. Sarà. 1999. The Role of Sponges in the Terra Nova Bay Ecosystem, p. 539-549. In: F. Faranda (Ed.). Benthic Ecology of Terra Nova Bay. Berlin, Springer-Verlag, 549 p.

Cuartas, E.I. 1986. Poríferos de la Campaña del B/I “Dr. E. Holmberg" (Demospongiae). Neotropica 32: 45-48.

Cuartas, E.I. 1992. Poríferos de la provincia biogeográfica argentina. III. Poecilosclerida (Demospongiae) del litoral marplatense. Physis, Secc. A 47 (113): 73-88.

Desqueyroux, R. 1972. Gubki (Porifera) sobranie u Iuynix Shetlandskix Ostrovof y Antarktichescovo poluostrovo. Issledovania Fauni Morei 11 (19): 49-55.

Desqueyroux, R. 1975. Esponjas (Porifera) de la region antartica chilena. Cahiers de Biologie Marine 16: 47-82.

Desqueyroux, R. 1976. Démosponges signalées pour la région dês Canaux du Chili. Cahiers de Biologie Marine 17: 93-109.

Desqueyroux, R. \& H. Moyano. 1987. Zoogeografia de Demospongias chilenas. Boletin de la Sociedad de Biologia de Concepción 58: 39-66.

Desqueyroux-Faúndez, R. 1989. Demospongiae (Porifera) del litoral chileno antártico. Serie Científica del Instituto Antártico Chileno 39: 97-158.

Desqueyroux-Faúndez, R. \& R.W.M. Van Soest. 1996. A review of Iophonidae, Myxillidae and Tedaniidae ocurring in the South East Pacific, (Porifera: Poecilosclerida). Revue Suisse de Zoologie 103 (1): 3-79.

Gaino, E.; G. Bavestrello; R. Cattaneo-Vietti \& M. Sarà. 1994. Scanning electron microscope evidence for diatom uptake by two Antarctic sponges. Polar Biology 14: 55-58.

GutT, J. \& W.M. Koltun. 1995. Sponges of the Lazarev and Weddell Sea, Antarctica: explanations for their patchy occurrence. Antarctic Science 7 (3): 227-234.

Hentschel, E. 1914. Monaxone Kieselschwämme und Hornschwämme der Deutschen Südpolar-Expedition 1901-1903. Deutsche Südpolar-Expedition, 1901-1903 15 (1): 35-141.

Hooper, J.N.A. 2002. Family Acarnidae, p. 412-431. In: J.N.A. Hooper \& R.W.M. Van Soest (Eds). Systema Porifera: a guide to the classification of sponges. New York, Kluwer Academic, vol. 1, XIX+1101p.

Jayatilake, G.S.; B.J. Baker \& J.B. McClintock. 1995. Isolation and identification of a stilbene derivative from the Antarctic sponge Kirkpatrickia variolosa. Journal of Natural Products 58 (12): 1958-1960.

KirKPatrick, R. 1907. Preliminary report on the Monaxonellida of the National Antarctic Expedition. Annals and Magazine of Natural History (7) 20 (117): 271-291.

KirkPatrick, R. 1908. Porifera (Sponges). II. Tetraxonida, Dendy. National Antarctic Expedition 1901-1904 4: 1-56.

Koltun, W.M. 1964. Sponges of the Antarctic. I. Tetraxonida and Cornacuspongida. Biological Reports of the Soviet Antarctic Expedition (1955-1958). Issledovania Fauni Morei 2: 6-116.
Koltun, W.M. 1976. Porifera - Part I: Antarctic Sponges. B.A.N.Z. Antarctic Research Expedition, Reports, Series B, Zoology and Botany, 9 (4): 147-198.

LÉvi, C. 1956. Éponges littorales des Iles Kerguelen récoltées par M. Angot. Mémoires de L'Institute Scientifique de Madagascar, Série A, Biologie Animale 10: 25-34.

LÉvi, C. 1964. Éponges de la Baie du Morbihan (Iles de Kerguelen). Comité National Français des Rechearches Antarctiques 1 (8): 149-155.

McClintock, J.B. \& J.J. Gautier. 1992. Antimicrobial activities of Antarctic sponges. Antarctic Science 4 (2): 179-183.

Mothes, B. \& C.B. Lerner. 1995. Ectyonanchora ruthae sp. n. (Myxillidae) e outras esponjas detectadas na $1^{\mathrm{a}}$ Expedição Antártica Brasileira (Porifera: Hexactinellida e Demospongiae). Biociências 3 (1): 155-171.

Mothes, B.; M.A. Campos; C.B. Lerner \& Ferreira-Correia, M.M. 2004a. Esponjas (Demospongiae, Halichondrida) da costa do Maranhão, Brasil. Iheringia, Série Zoologia 94 (2): 149-154.

Mothes, B.; R.R. Capítoli; C. Lerner \& M.A. Campos. 2004b. Filo Porifera, p. 57-63. In: A.C.Z. Amaral \& C.L.D.B. RossiWongtschowski (Eds). Biodiversidade Bentônica da Região Sudeste-Sul do Brasil - Plataforma Externa e talude Superior. São Paulo, Instituto Oceanográfico, USP, 216p.

Mothes-de-Moraes, B. 1978. Esponjas tetraxonidas do litoral sulbrasileiro: II. Material coletado pelo N/Oc. "Prof. W. Besnard" durante o programa RS. Boletim do Instituto de Oceanografia 27 (2): 57-78.

Pansini, M; B. Calcinai; R. Cattaneo-Vietti \& M. Sarà. 1994. Demosponges from Terra Nova bay (Ross Sea, Antarctica): 1987/88 and 1989/89 P.N.R.A. Expeditions. National Scientific Comission for Antarctica 3: 67-100.

PAnSINI, M. \& M. SARÀ. 1999. Taxonomical and biogeographical notes on the sponges of the Straits of Magellan, p. 203-208. In: W.E. Arntz \& C. Ríos (Eds). Magellan-Antarctic: Ecosystems that drifted apart. Scientia Marina, Barcelona, 63 (Suppl. 1), 518p.

Perry, N.B.; L. Ettouati; M. Litaudon; J.W. Blunt; M.H.G. Munro; S. Parkin \& H. Hope. 1994. Alkaloids from the Antarctic Sponge Kirkpatrickia variolosa. Part 1: variolin B, a New Antitumor and Antiviral Compound. Tetrahedron 50 (13): 3987-3992.

Ridley, S.O. \& A. Dendy. 1886. Preliminary Report on the Monaxonida collected by H.M.S. 'Challenger'. Annals and Magazine of Natural History (5) 18 (107): 325-351, 470-493.

Ridley, S.O. \& A. Dendy. 1887. Report on the Monaxonida collected by H.M.S. 'Challenger' during the years 1873-1876. Report on the Scientific Results of the Voyage of H.M.S. 'Challenger' 1873-1876, Zoology 20: 1-279.

Ríos, P.; F.J. CRISTobo \& V. Urgorri. 2004. Poecilosclerida (Porifera, Demospongiae) collected by the spanish antarctic expedition BENTART-94. Cahiers de Biologie Marine 45: 97-119.

Samait, T.; M.J. Gibbons; M. Kelly \& M. Davies-Koleman. 2003. South African Latrunculiidae (Porifera: Demospongiae: 
Poecilosclerida): descriptions of new species of Latrunculia du Bocage, Strongylodesma Lévi, and Tsitsikamma Samaai \& Kelly. Zootaxa 371: 1-26.

Samaai, T.; M.J. Gibbons \& M. Kelly. 2006. Revision of the genus Latrunculia du Bocage, 1869 (Porifera: Demospongiae: Latrunculiidae) with descriptions of new species from New Caledonia and the Northeastern Pacific. Zootaxa 1127: 1-71.

SARÀ, M. 1978. Demospongiae di acque superficiale della Terra del Fuoco (Spedizioni AMF Mares - GRSTS e SAI). Bollettino dei Musei e degli Instituti Biologici dell’Universitá di Genova 46: 7-117.

SARÀ, M. 1991. Contribution to the knowledge of the Porifera from the Strait of Magellan. Memorie di Biologia Marina e di Oceanografia 19: 233-235.

Sarà, M.; A. Balduzzi; M. Barbieri; G. Bavestrello; C.N. Bianchi; F. Boero; R. Cattaneo-Vietti; G. Corriero; C. Morri \& M. Pansini. 1990. Hard bottom zoobenthos: an analysis of its composition, distribution and of the adaptative strategies of the species. National Scientific Comission for Antarctica 2: 249-260.

SiLvA, C.M.M. \& B. Mothes. 1996. SEM analysis: an important instrument in the study of marine sponges biodiversity. Acta Microscopica 5 (B): 188-189.

Topsent, E. 1907. Poecilosclérides nouvelles recueillies par le Français dans l'Antarctique. Bulletin du Muséum National
d'Histoire Naturelle 13: 69-76.

Topsent, E. 1908. Spongiaires. Expédition Antarctique Française (1903-1905) commandée par le Dr. Jean Charcot 4: 1-37.

Topsent, E. 1913. Spongiaires de l'Expédition Antarctique Nationale écossaise. Transactions of the Royal Society of Edinburgh 49 (3): 579-643.

Topsent, E. 1915. Spongiaires recueillies par la Scotia dans l'Antarctique (1903-1904). Transactions of the Royal Society of Edinburgh 51 (Suppl.): 35-43.

Topsent, E. 1916. Diagnoses d'Eponges recueillies dans l'Antarctique par le Pourquoi pas?. Bulletin du Muséum National d'Histoire Naturelle (1) 22 (3): 163-172.

Topsent, E. 1917. Spongiaires. Deuxième Expédition Antarctique Française (1908-1910) commandée par le Dr. Jean Charcot. Paris, Masson, 88p.

Trimurtulu, G.; D.J. FaulKner; N.B. Perry; L. Ettouati; M. Litaudon; J.W. Blunt; M.H.G. Munro \& G.B. Jameson. 1994. Alkaloids from the Antarctic sponge Kirkpatrickia variolosa. Part 2: Variolin A and $N\left(3^{\prime}\right)$-methyl tetrahydrovariolin B. Tetrahedron 50 (13): 3993-4000.

VACELET, J. \& F. ARnAud. 1972. Invertébrés Marins des XIIème et XVème Expéditions Antarctiques Françaises en Terre Adélie. 2. Démosponges. Tethys Supplement 4: 9-24.

Received in 01.XII.2006; accepted in 23.VIII.2007. 